\title{
Data driven modeling of plastic deformation
}

\author{
Daniele Versino $^{\mathrm{a}, *}$, Tonda Alberto ${ }^{\mathrm{b}, * *}$, Curt A. Bronkhorst ${ }^{\mathrm{a}}$ \\ ${ }^{a}$ Theoretical Division, T-3, Los Alamos National Laboratory \\ Los Alamos, New Mexico 87545, USA \\ ${ }^{b}$ UMR GMPA, AgroParisTech, INRA, Université Paris-Saclay \\ 1 av. Brétignières, 78850, Thiverval-Grignon, France
}

\begin{abstract}
In this paper the application of machine learning techniques for the development of constitutive material models is being investigated. A flow stress model, for strain rates ranging from $10^{-4}$ to $10^{12}$ (quasi-static to highly dynamic), and temperatures ranging from room temperature to over $1000 \mathrm{~K}$, is obtained by beginning directly with experimental stress-strain data for Copper. An incrementally objective and fully implicit time integration scheme is employed to integrate the hypo-elastic constitutive model, which is then implemented into a finite element code for evaluation. Accuracy and performance of the flow stress models derived from symbolic regression are assessed by comparison to Taylor anvil impact data. The results obtained with the free-form constitutive material model are compared to well-established strength models such as the Preston-Tonks-Wallace (PTW) model and the Mechanical Threshold Stress (MTS) model. Preliminary results show candidate free-form models comparing well with data in regions of stress-strain space with sufficient experimental data, pointing to a potential means for both rapid prototyping in future model development, as well as the use of machine learning in capturing more data as a guide for more advanced model development.
\end{abstract}

Keywords: Strength models, high strain rate, $\mathrm{J}_{2}$ plasticity, machine learning, symbolic regression, Taylor anvil impact

\section{Introduction}

Modeling materials is of extreme importance for a wide range of practical reasons: for example, since experiments are costly and time-consuming to perform, a mathematical model can provide fast predictions of material behavior, allowing experts to test new structures or solutions in an inexpensive way. Researchers in the past focused on the development of new phenomenological constitutive models by finding approximate expressions that described the physical phenomena underlying the observed macroscale behavior. These expressions usually depended on several empirical parameters that were computed, or calibrated, using experimental data. Stripped to the essential, material modeling at the macroscale level consists in finding the best fit (equation) for a set of experimental data, using observations at micro and macro scales. As models become more complex and advanced in their physical basis, however, there is a corresponding rise in the complexity of empirical expressions and increased difficulty in the parameter calibration. This complexity is eased through a focus on the development of more physically based models where quantities are well defined and can be evaluated independently.

From a different perspective, developing a material model at the macroscopic continuum scale can be seen as an optimization problem: for a given set of points in an $n$-dimensional space, find the functional (or set thereof) that is closest to the input points. The complexity of such an optimization problem is non-polynomial (NP-hard problem) [1]. If the form of the interpolating function is an a priori, the problem reduces to finding the parameter values that

*Daniele Versino, Tel: +1 5056672137

** Alberto Tonda, Tel: +33130814596.

Email addresses: daniele.versino@lanl.gov (Daniele Versino), alberto.tonda@grignon.inra.fr (Tonda Alberto) 
produce the optimal fit for the dataset, which can be solved with polynomial complexity (i.e. the mean least square approximation is probably the simplest example of this category). In the past, limited computational resource and lack of appropriate solution algorithms rendered the NP-hard approach intractable. Nonetheless, current advances in machine learning (ML) techniques and increased computational power make the methodology with non-polynomial complexity appealing. Several solutions for NP-hard optimization problems resort to stochastic techniques to find reasonable, approximate solutions to the global optimum.

The current paper explores the application of stochastic techniques to the modeling of plastic deformation in polycrystalline metallic materials, specifically within a $\mathbf{J}_{2}$ framework with a flow potential surface. Over the broad range of conditions of interest for this class of materials, the ability of the material to accommodate deformation while avoiding material failure is determined by the availability of the mechanisms of dislocation glide and deformation twinning [2-5]. Each of these is driven by atom level processes involving the motion of dislocations due to the imposition of stress on the material to drive the motion, multiplication, and interaction of dislocations and mechanical twins which result in an orientational transformation. This plastic deformation if extended to high levels causes local rotations in the material's single crystals and both morphological and crystallographic texture is developed [6]. These physical events taking place in the material are in general very much sensitive to the state conditions (e.g. temperature, strain rate) and deformation history of the material. In addition, plasticity processes are also very much impacted by the local structure of the material such are material type, grain size, and chemical composition [7, 8]. Developing models to accurately represent these complex dependencies for a very broad range of state conditions and deformation histories is still very much a topic of research $[9,10]$. In general, models are available in a broad range of complexities and employ many different functional forms from the very simple which have little physical basis in the choice of internal state variables (which are very easy to use but are less accurate) to very sophisticated which much more accurately represent the basic physical processes facilitating plastic deformation (but may require a specialist to understand and use). It is still generally a challenge to strike the proper balance between ease of use and physical accuracy. In the future, this balance can be struck much more efficiently if specific models are developed for specific materials and conditions rather than universally applicable models as we practice today which compromise accuracy for universality. As such, new machine learning tools can assist in postulating the essential mathematical form of a model for the specific material and conditions of interest.

As the complexity of $\mathbf{J}_{2}$ based material strength models is increased, the evaluation of their parameters for a target material became an increasingly long and expensive process, requiring extra experiments. It is not surprising that the machine learning community tried to tackle the issue with black-box models, automatically derived from experimental data. Artificial Neural Networks (ANN) are surely among the most popular solutions, for their great effectiveness and ease of use: for example, in [11] ANNs are trained to predict flow stress for 7107 aluminum alloy, showing a good result when compared to the classical Johnson-Cook model; in [12], ANNs obtained a favorable comparison to ZerilliArmstrong and Arrhenius-type models for high-temperature deformation in T24 steel; and in [13], a two-layer ANN is used to learn flow stress dynamics in 5086 aluminum alloy. In [14] another ML technique, Support Vector Machines Regression (SVRs), is used to find a model for flow stress in Austenitic Stainless Steel 304, returning good results especially for high temperatures, even when compared to ANNs trained on the same dataset; however, SVR requires the precise tuning of several meta-parameters and an appropriate choice of the kernel, in order to be effective.

While ML techniques can be successful, in order to capture the full dynamics of a phenomenon, they usually require a considerable amount of experimental data, that can be costly or simply unpractical to produce. For this reason, works with ANNs found in literature are usually limited to a relatively small range of values for each meaningful variable (temperature, strain, strain rate). There are, however, ML techniques that can deliver satisfying results starting from a limited amount of data points, over a much wider range of values.

The ML technique of choice for the problem in study is a stochastic technique called symbolic regression. The inputs required by this methodology are the dataset and a group of mathematical operators and functions that can be used to fit the data. The output is a set of equations, with their associated complexity, that best describe (fit) the experimental data. This methodology is preferable to a black-box model; it satisfies the researcher's need of observing the relation between the variables of the problem, as well as providing advantages in the implementation phase. Obtaining an equation for the strength model, and hence its analytic derivative, allows the use of iterative solvers that employ jacobians (i.e. Newton-Raphson scheme) which afford higher convergence order.

Material data covering a broad range of temperatures and strain rates are difficult to find in literature. Copper is one of the most extensively characterized materials and hence it is used as a proof of concept in the present paper. 


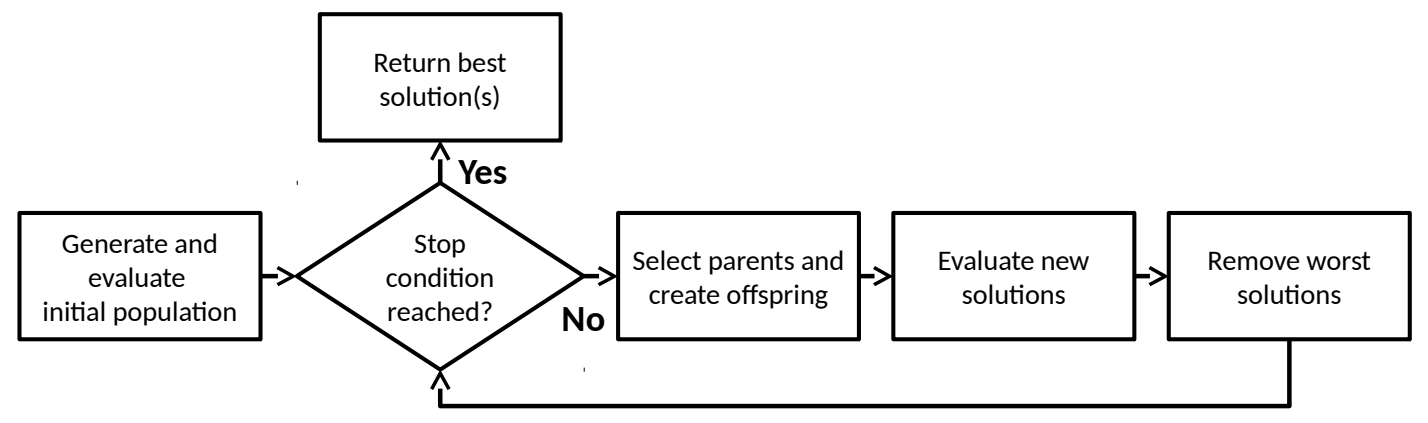

Figure 1: Flowchart of a classical EA: the initial candidate solutions are randomly generated and after evaluating their fitness the best individuals are used/mutated to generate new solutions. The worst individuals are removed from the genetic pool and the process is iterated until a stop condition is reached.

Although the obtained strength models will be specific for copper, the general methodology can be applied to any material of interest, provided that enough data are available. Once a strength model is generated, it is implemented into a commercial finite element code using a $\mathbf{J}_{2}$ radial return algorithm, and a standard implicit incrementally objective time integration scheme is then employed. Accuracy of the strength models is assessed by means of numerical simulations of Taylor anvil impacts [15]. The numerical simulation is characterized by high strain-rates and large plastic deformations that cause an increase of the sample's temperature. Results of the strength models generated through symbolic regression are then compared to established models, Mechanical Threshold Strength (MTS) and Preston-Tonks-Wallace (PTW), and to the available experimental results. In the present work, for simplicity, data used by the symbolic regression have physical dimensions. While recognizing the importance of scale invariance in the development of material models [16], nondimensionalization can be obtained by straightforward application of the Buckingham $\Pi$ theorem [17] and it is not explored in the current work.

The paper is structured by introducing the symbolic regression technique in Section 2. Two available strength models for high strain rates and temperatures are presented in Section 3. In Section 4, the experimental stress-strain curves of copper are used to derive, through symbolic regression (SR), four new strength models. The numerical implementation of MTS, PTW and the SR models is detailed in Section 5 and assessment of the data-driven constitutive models is carried out in Section 6. Finally, a summary and concluding remarks are presented in Section 7.

\section{Symbolic regression}

Symbolic regression is a machine learning technique from the field of evolutionary computation [18], able to extract free-form equations that correlate with data from a given experimental dataset. Evolutionary algorithms (EAs) are stochastic optimization techniques that loosely mimic natural evolution. Generally speaking, an EA starts by creating a set of random candidate solutions called population. Each candidate solution, termed individual, is then evaluated with regards to an objective function, called fitness. The best solutions have a higher probability to be selected for creating offspring, that are, randomly mutated versions of the original individuals. Offspring are then evaluated, and added to the population. At the end of each iteration, the worst individuals in the population are removed. Then, new offspring are produced, and the algorithm continues until a user-defined stop condition is reached (e.g. wall-clock time limit, satisfying value of the best candidate solution, etc.). A flowchart for a generic EA is presented in Figure 1.

The original idea for symbolic regression was presented in [1], and it was subsequently popularized by the commercial tool Eureqa Formulize $e^{1}$ [19]. In this EA, candidate solutions are encoded as trees, with terminal nodes

\footnotetext{
${ }^{1}$ http://nutonian.com/
} 
corresponding to constants and variables of the problem, while intermediate nodes encode mathematical functions such as $\{+,-, *, /, \ldots\}$. All the nodes are collectively termed building blocks, and are user-defined. The fitness function is usually proportional to the absolute or squared error between experimental data and values predicted by a candidate solution, with parsimony corrections to favor more compact equations. An example of individual for a symbolic regression problem is presented in Figure 2.
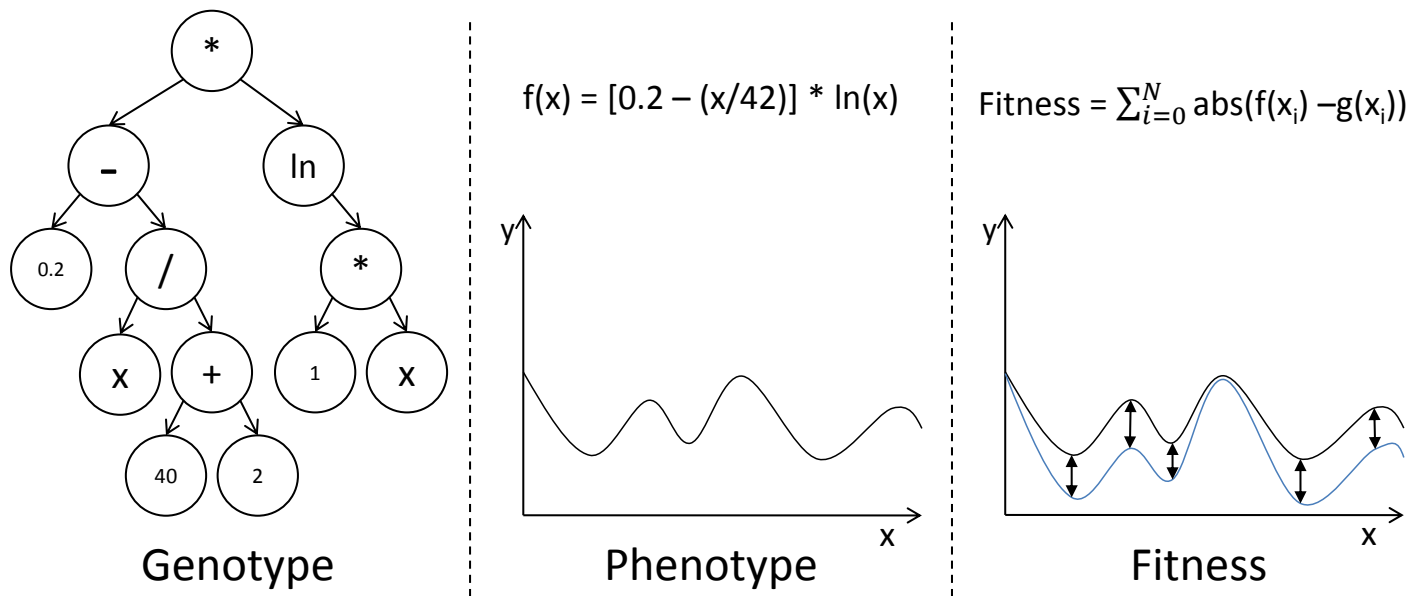

Figure 2: A candidate solution in a typical symbolic regression problem. Following the natural evolution metaphor, the internal representation (a binary tree) is termed genotype. The phenotype is the corresponding function, while the fitness to minimize is usually the absolute or squared error with respect to experimental points.

An additional advantage of this technique is the delivery of human-readable models. While ANN and SVM models are usually hard to make sense of, information can always be extracted from equations, even when they are extremely complex: a human expert could use automatically generated results to infer properties of the target phenomenon, and eventually use them as a base to build a better model. As symbolic regression explores the vast search space of all possible equations, the real-valued parameters of the obtained models can often be further optimized resorting to classical techniques such as gradient descent, thus improving their overall fitting. In this work, however, we intentionally skip this passage, in order to better evaluate the potential of this machine learning algorithm as it is.

Modern approaches to symbolic regression make use of sophisticated techniques to speed up convergence and improve the quality of results: competitive co-evolution [20], incorporation of expert knowledge [21], noise modeling [22], and, most notably, Pareto-like optimization [23]. Taking inspiration from works on multi-objective optimization, Pareto-like symbolic regression evaluates candidate solutions on more than one feature, for example fitting and complexity: instead of returning the user a single, optimal solution, such algorithms will show a Pareto front comprising several solutions, each one an optimal trade-off between the two objectives.

It is important to notice that, being a stochastic optimization process, there is no guarantee that an EA will find the global optimum for a given problem: while there are proofs of convergence for simple case studies [24], when the structure of a candidate solution is too complex, formally proving properties has so far been unfeasible. Nevertheless, EAs are routinely used for real-world NP-hard problems where classical optimization techniques fail, or take too much time to converge [25-27].

\section{Existing strength models}

Strength models developed over the past forty years for the modeling of plastic flow at high temperatures and strain rates, may be classified into two categories: purely empirical models and physically based models. To the first category belong the Johnson-Cook model [28] and the Steinberg-Cochran-Guinan-Lund model [29, 30] which show unrealistic strain-rate independence at high strain rates or for any strain rate respectively. To the second category belong models that account for dislocation interactions and dynamics. Among these models the Zerilli-Armstrong 
model [31], the Mechanical Threshold Stress (MTS) model [32] and the Preston-Tonks-Wallace (PTW) model [16] are widely used and reported.

Hereinafter, the two latter strength models are briefly summarized as they will later be used as a comparison for the results obtained through symbolic regression. For the considered material, copper, the values of the parameters used by MTS and PTW are given in [33] and [16], respectively.

\subsection{Mechanical Threshold Strength}

The mechanical threshold strength (MTS) model [32, 34-36] characterizes the flow stress of material by considering the combined effect of different sources of resistances to dislocation motion. The interdiction of dislocations is managed through short-range barriers, that can be surpassed thanks to thermal activation; and long-range barriers, which are unaffected by thermal energy. This model can be seen as a complex, non-linear isotropic hardening law, which accounts for strain hardening, strain-rate hardening, and thermal softening effects.

According to MTS, the flow stress $\tau$ of a material can be expressed as:

$$
\tau=\tau_{a}+\frac{G(\mathrm{~T})}{G_{0}}\left[S_{i}\left(\mathrm{~T}, \dot{\varepsilon}^{p}\right) \tau_{i}+S_{\xi}\left(\mathrm{T}, \dot{\varepsilon}^{p}\right) \tau_{\xi}+S_{s}\left(\mathrm{~T}, \dot{\varepsilon}^{p}\right) \tau_{s}\right]
$$

where $\tau_{a}$ is the constant athermal component of the flow stress, $\tau_{i}$ is the constant intrinsic lattice resistance at $\mathrm{T}=0 \mathrm{~K}$, and $\tau_{\xi}$ represents the evolving flow resistance at $\mathrm{T}=0 \mathrm{~K}$, due to dislocation structure. The flow stress term dependent on the thermally related solute diffusion, $\tau_{s}$, appearing in the most general MTS form, is neglected in the present work. $S_{i}$ and $S_{\xi}$ introduce rate and temperature kinetics, while the temperature-dependent shear modulus is $G$, and its value at $\mathrm{T}=0 \mathrm{~K}$ is $G_{0}$. The shear modulus is given by the following empirical expression:

$$
G(\mathrm{~T})=G_{0}-\frac{D_{0}}{\exp \left(\frac{\mathrm{T}_{0}}{\mathrm{~T}}\right)-1},
$$

where $\mathrm{T}_{0}$ is a reference temperature. Term $S_{i}$ is expressed as:

$$
S_{i}\left(\mathrm{~T}, \dot{\varepsilon}^{p}\right)=\left(1-\left[\frac{k \mathrm{~T}}{G b^{3} g_{0 i}} \ln \frac{\dot{\varepsilon}_{0 i}^{p}}{\dot{\varepsilon}^{p}}\right]^{\frac{1}{q_{i}}}\right)^{\frac{1}{p_{i}}}
$$

where $k$ is Boltzmann's constant, $b$ is the magnitude of the Burgers vector, $g_{0 i}$ is a normalized activation energy, $\dot{\varepsilon}_{0 i}^{p}$ is a reference strain rate, $p_{i} \in[0,1]$ and $q_{i} \in[0,1]$ are exponents that define the shape of the energy barrier profile. Term $S_{\xi}$ is given by a similar expression, with corresponding constants:

$$
S_{\xi}\left(\mathrm{T}, \dot{\varepsilon}^{p}\right)=\left(1-\left[\frac{k \mathrm{~T}}{G b^{3} g_{0 \xi}} \ln \frac{\dot{\varepsilon}_{0 \xi}^{p}}{\dot{\varepsilon}^{p}}\right]^{\frac{1}{q_{\xi}}}\right)^{\frac{1}{p_{\xi}}} .
$$

Finally, the mechanical threshold $\tau_{\varepsilon}$, reflecting the evolution of the dislocation structure, evolves as

$$
\frac{d \tau_{\varepsilon}}{d \dot{\varepsilon}^{p}}=h_{0}\left(\dot{\varepsilon}^{p}, \mathrm{~T}\right)\left(1-\frac{\tau_{\varepsilon}}{\tau_{\xi S}}\right)^{K},
$$

where the saturation threshold stress, $\tau_{\varepsilon S}$, is

$$
\tau_{\varepsilon S}=\tau_{\varepsilon S 0}\left(\frac{\dot{\varepsilon}^{p}}{\dot{\varepsilon}_{0 \varepsilon S}^{p}}\right)^{\frac{k T}{G b^{3} g_{0 \varepsilon S}}},
$$

and where $g_{0 \varepsilon S}$ is the normalized energy of activation, while $\dot{\varepsilon}_{0 \varepsilon S}^{p}$ is a reference strain rate. 


\subsection{Preston-Tonks-Wallace}

The Preston-Tonks-Wallace (PTW) model [16] describes the plastic flow stress $\tau$ as a function of the amount of strain $\varepsilon^{p}$ it has undergone, the strain rate $\dot{\varepsilon}^{p}$, the material temperature $\mathrm{T}$ and its density $\rho$. The model assumes that the plastic flow stress is independent of the history of the material (other than plastic strain), and that the plastic flow is isotropic. The PTW model makes use of three scaled, dimensionless variables:

$$
\bar{\tau}=\frac{\tau}{G(\rho, \mathrm{T})},
$$

where $\tau$ is the flow stress, defined as $\tau=\sigma / 2$, where $\sigma$ is the Von Mises equivalent deviatoric stress; and $G(\rho, \mathrm{T})$ is the shear modulus, function of material density and temperature. Temperature is scaled according to:

$$
\overline{\mathrm{T}}=\frac{\mathrm{T}}{\mathrm{T}_{m}(\rho)},
$$

where $\mathrm{T}_{m}$ is the melt temperature of the material, function of its density. Finally, $\dot{\varepsilon}^{p}$ is scaled to an appropriate rate:

$$
\dot{\xi}(\rho, \overline{\mathrm{T}})=\frac{1}{2}\left(\frac{4 \pi \rho}{3 M}\right)^{\frac{1}{3}}\left(\frac{G}{\rho}\right)^{\frac{1}{2}},
$$

where $M$ is the atomic mass of the material. The strain rate in the PTW model will always appear as $\dot{\varepsilon}^{p} / \dot{\xi}$. The shear modulus $G$ is:

$$
G(\rho, \overline{\mathrm{T}})=G_{0}(\rho)(1-\alpha \overline{\mathrm{T}}),
$$

where $G_{0}(\rho)$ is the shear modulus at $\mathrm{T}=0$ and $\alpha>0$ is a parameter dependent on the material.

The scaled stress $\bar{\tau}$ ranges between the yield stress $\bar{\tau}_{y}$ and a saturation value $\bar{\tau}_{s}$, with a functional form dependent on the strain $\varepsilon^{p}$ :

$$
\bar{\tau}=\bar{\tau}_{s}+\frac{1}{p}\left(s_{0}-\bar{\tau}_{y}\right) \ln \left\{1-\left[1-\exp \left(-p \frac{\bar{\tau}_{s}-\bar{\tau}_{y}}{s_{0}-\bar{\tau}_{y}}\right)\right] \exp \left[-\frac{p \theta \varepsilon^{p}}{\left(s_{0}-\bar{\tau}_{y}\right)\left[\exp \left(p \frac{\bar{\tau}_{s}-\bar{\tau}_{y}}{s_{0}-\bar{\tau}_{y}}\right)-1\right]}\right]\right\},
$$

where $p$ and $\theta$ are material-dependent parameters, and $s_{0}$ is the value that $\bar{\tau}_{s}$ assumes at zero temperature. The values for $\bar{\tau}_{y}$ and $\bar{\tau}_{s}$ are given by:

$$
\bar{\tau}_{y}^{L}=y_{0}-\left(y_{0}-y_{\infty}\right) \operatorname{erf}\left[\kappa \overline{\mathrm{T}} \ln \left(\frac{\gamma \dot{\xi}}{\dot{\varepsilon}^{p}}\right)\right], \quad \quad \bar{\tau}_{s}^{L}=s_{0}-\left(s_{0}-s_{\infty}\right) \operatorname{erf}\left[\kappa \overline{\mathrm{T}} \ln \left(\frac{\gamma \dot{\xi}}{\dot{\varepsilon}^{p}}\right)\right],
$$

where $\kappa$ and $\gamma$ are dimensionless material parameters and erf denotes the error function. Furthermore, $L, M$ and $H$ superscripts are employed to indicate respectively low $\left(\dot{\varepsilon}^{p}<10^{4}\right)$, intermediate $\left(10^{4} \leq \dot{\varepsilon}^{p}<10^{9}\right)$ and high strain rates $\left(\dot{\varepsilon}^{p} \geq 10^{9}\right)$. The parameters $y_{0}$ and $y_{\infty}$ are the values that $\bar{\tau}_{y}$ takes at zero temperature and very high temperatures, respectively; the same holds for $s_{0}$ and $s_{\infty}$ with respect to $\bar{\tau}_{s}$.

When the strain rates become very high $\left(\dot{\varepsilon}^{p}>10^{8}\right)$ the plastic deformation process can be appropriately described by Wallace's theory of overdriven shocks in metals [37], so that the saturation stress becomes

$$
\bar{\tau}_{s}^{H}=s_{0}\left(\frac{\dot{\varepsilon}^{p}}{\gamma \dot{\xi}}\right)^{\beta},
$$

where the transition between low and high strain rates accounted for by

$$
\bar{\tau}_{s}=\max \left\{\bar{\tau}_{s}^{L}, \bar{\tau}_{s}^{H}\right\} .
$$


At very high strain rate the value of yield stress is the same as the saturation stress, that is, $\bar{\tau}_{y}^{H}=\bar{\tau}_{s}^{H}$ whereas, for intermediate strain rates, a power-law is employed leading to

$$
\bar{\tau}_{y}^{M}=y_{1}\left(\frac{\dot{\varepsilon}^{p}}{\gamma \dot{\xi}}\right)^{y_{2}} .
$$

Analogously to the saturation stress, a transition between the three strain rate regimes is accounted for by introducing the following formula for the yield stress

$$
\bar{\tau}_{y}=\max \left\{\bar{\tau}_{y}^{L}, \min \left\{\bar{\tau}_{y}^{M}, \bar{\tau}_{y}^{H}\right\}\right\}
$$

\section{Symbolic Regression models}

In this section the strength models obtained through symbolic regression are summarized. Four models are derived using different datasets, with the addition of expert knowledge. Among the candidate models obtained, the ones that provide the overall best fitting are presented: the equations selected are close to the "knee" of their respective Pareto front, featuring a balance between complexity and fitting. Complexity is a measure of the computational time necessary to evaluate an expression and we chose the equation located at a "bending point" of the Pareto front, where complexity increases but the gain in fitting becomes less and less relevant. Finally, it is worth mentioning that points (candidate equations) on the Pareto front have different functional forms. For ease of reading, datasets and accuracy of the models with respect to the training data are collected in Appendix A. In the following, coherently with the other models previously presented, $\tau$ is the stress, $\varepsilon^{p}$ is the strain, $\dot{\varepsilon}^{p}$ is the strain rate, and $\mathrm{T}$ is the temperature. For convenience, $\hat{\tau}=\ln (\tau), \hat{\varepsilon}^{p}=\ln \left(\varepsilon^{p}\right), \hat{\dot{\varepsilon}}^{p}=\ln \left(\dot{\varepsilon}^{p}\right)$, and $\hat{\mathrm{T}}=\ln (\mathrm{T})$ variables are also introduced. Stress, temperature and plastic strain and strain rate are not normalized: the units of measure of $\tau, \dot{\varepsilon}^{p}$ and $\mathrm{T}$ are $\mathrm{MPa}, \mathrm{s}^{-1}$ and $\mathrm{K}$, respectively.

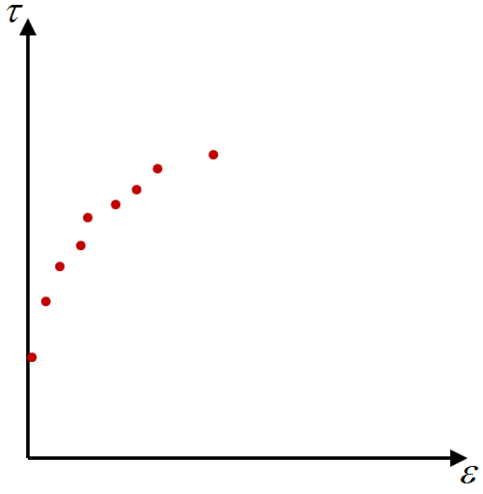

(a) Original data

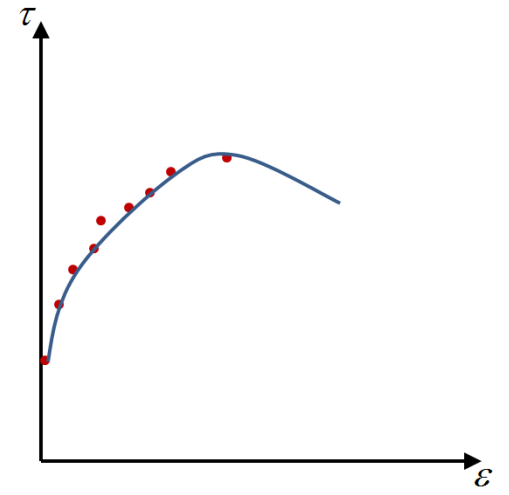

(b) Original data: $\tau=\tau(\varepsilon)$

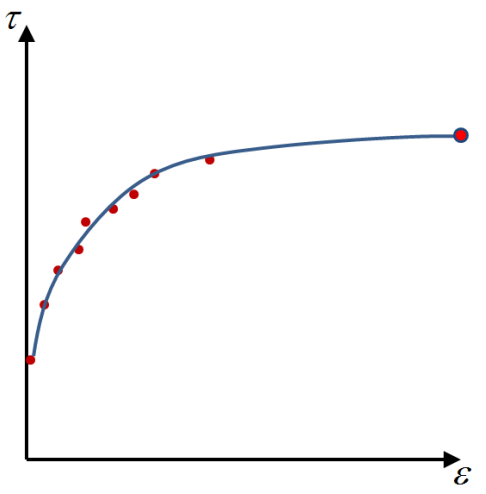

(c) Biased data: $\tau=\tau(\varepsilon)$

Figure 3: Derivation of a strength model from simple compression Hopkinson bar data (room temperature and strain rate $\approx 10^{3}$ ). The training data points, shown in 3a, are missing information for high levels of $\varepsilon$. Symbolic regression finds a model that fits the given points well enough, but has an incorrect behavior where data is missing, as expressed by the blue line in 3b. Adding an artificial point, taken from expert knowledge on the process, leads the machine learning algorithm towards a better model, as shown in $3 \mathrm{c}$.

\section{1. $S R-1$}

SR-1 indicates the best result of the very first, and naive, symbolic regression experiment on the D1, D2 and D3 datasets. The algorithm is set to find the strength model $\tau=f\left(\varepsilon^{p}, \dot{\varepsilon}^{p}, \mathrm{~T}\right)$. A first Pareto front returns an equation with good fitting and reasonable complexity, that is selected for further evaluation. The equation has the following form:

$$
\tau=6.025+0.0095 \mathrm{~T}+\varepsilon^{p}\left(3217.611+2314.333 \varepsilon^{p}-4514.091 \sqrt{\varepsilon^{p}}-317.766\left(0.5835 \varepsilon^{p}\right)^{\dot{\varepsilon}^{p}}-\mathrm{T}\right),
$$

SR-1 in Eq. (17) is obtained by the process that takes from Fig. 3a to Fig. 3b, and exactly like in Fig. 3b the curve presents a decreasing trend for high values of $\varepsilon$. Although softening can clearly occur during plastic deformation, the 
behavior described by SR-1 is an artifact, since the considered datasets can be fitted quite accurately with a polynomial expression, with a negative slope for $\varepsilon^{p}>0.4$. Lacking data for that interval, expert knowledge has to be introduced in order to remove the incorrect negative slope.

\section{2. $S R-2$}

As for many machine learning approaches, it is possible to ease (or bias) the algorithm's task by providing human expert knowledge. In the case of symbolic regression, expert knowledge can be added through several means, for example:

- by forcing the use of user-defined variables (i.e. $\left.\ln (\tau), \exp \left(\varepsilon^{p}\right), \ldots\right)$;

- by introducing weights for each line of the dataset, diversifying the importance of data points the algorithm should fit during the run;

- by adding extra points, artificially generated, where there is no experimental data but the user is confident in the form they should present;

- by forcing candidate solutions to be always above a certain value (e.g. always positive);

- by suggesting some initial solutions, in the form of equations, that the algorithm will use to seed the initial population, and thus quickly driving the symbolic regression process towards specific areas of the search space.

During this second batch of ML experiments, all options for introducing expert knowledge are evaluated, with different outcomes. In the end, the most effective options seem to be the addition of artificial points, forcing the function to be non-negative, adding user-defined variables, and introduction of weights, in order to give each curve in the dataset the same importance, independently from their sampling density. The required functional form of the strength model is

$$
\tau=\frac{G(\mathrm{~T})}{G_{0}} e^{\hat{\tau}}
$$

with $\hat{\tau}=f\left(\hat{\varepsilon}^{p}, \hat{\dot{\varepsilon}}^{p}, \hat{\mathrm{T}}\right)$. In analogy with MTS and PTW, the $\frac{G(\mathrm{~T})}{G_{0}}$ term in Eq. (18) is introduced to exploit the known relation between shear modulus and temperature. The equation for $G(\mathrm{~T})$ is Eq. (2), the same used by MTS. Finally, the best model for $\hat{\tau}$ is given by

$$
\begin{aligned}
\hat{\tau} & =6.529+0.315 \min \left(\left[0.315, \hat{\varepsilon}^{p}\right]\right)+1.235 \cdot 10^{-5} \hat{\dot{\varepsilon}}^{p} \hat{\mathrm{T}} \max \left(\left[\hat{\dot{\varepsilon}}^{p}, 6.529\right]\right) \\
& -\hat{\varepsilon}^{p} \min \left(\left[0.058 \min \left(\left[0.315, \hat{\varepsilon}^{p}\right]\right), 1.235 \cdot 10^{-5} \hat{\dot{\varepsilon}}^{p} \hat{\mathrm{T}}\right]\right)-0.0097 \hat{\dot{\varepsilon}}^{p} \\
& -\hat{\mathrm{T}}\left(0.0021+0.001 \hat{\mathrm{T}} \tanh \left(\min \left(\left[-0.183, \hat{\varepsilon}^{p}\right]\right)\right)\right) .
\end{aligned}
$$

The process that leads to SR-2 in Eq. (18) can be visually represented by Figures 3a- 3c. The experimental data, datasets D1 to D5, are enriched with additional points, obtained from expert knowledge of the physical phenomenon, to inform the machine learning algorithm on the monotonicity of $\tau$. Moreover, using $e^{\hat{\tau}}$ ensures that the flow stress is always positive.

\section{3. $S R-3$}

Symbolic regression can also be used to learn derivatives. In order to simplify the algorithm's task, the interpolation of the shape of the $\tau-\varepsilon^{p}$ curve is separated from the computation of its asymptotic value. In two separate runs, the symbolic regression algorithm is asked to search for

$$
\frac{d \tau}{d \varepsilon^{p}}=f\left(\varepsilon^{p}, \dot{\varepsilon}^{p}, \hat{\dot{\varepsilon}}^{p}, \mathrm{~T}\right)
$$

and then

$$
\tau_{c}=g\left(\dot{\varepsilon}^{p}, \hat{\dot{\varepsilon}}^{p}, \mathrm{~T}\right),
$$




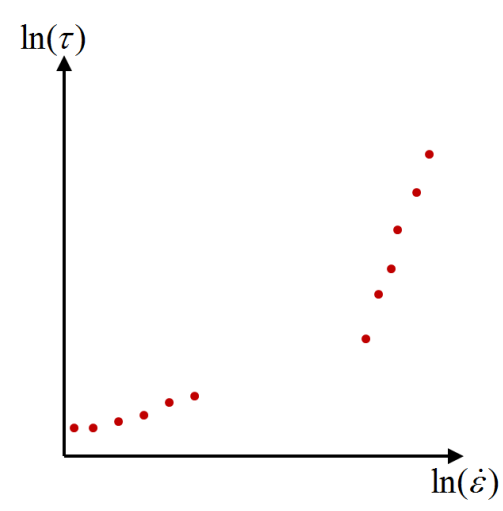

(a) Original data

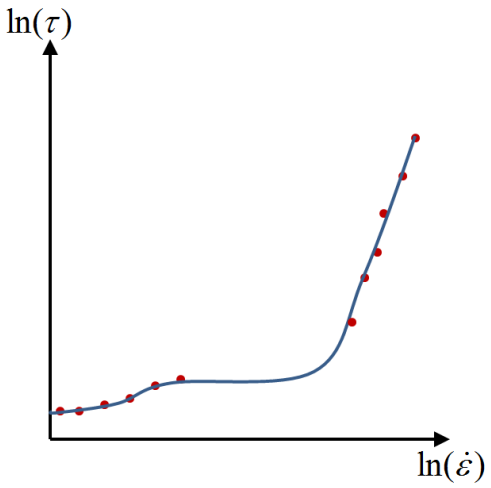

(b) Original data: $\tau=\tau(\dot{\varepsilon})$

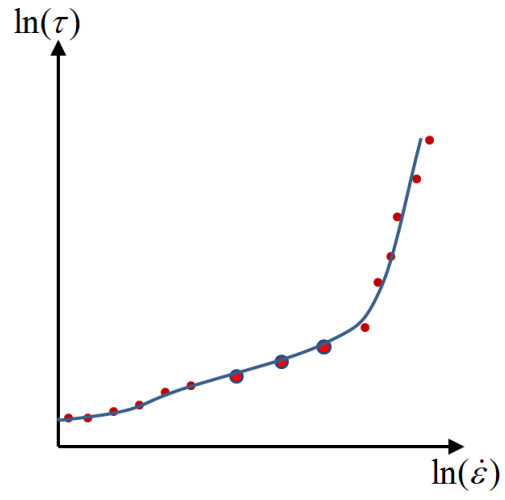

(c) Biased data: $\tau=\tau(\dot{\varepsilon})$

Figure 4: From experimental data to a saturation stress model (room temperature). Missing training points in the middle of the process, portrayed in $4 \mathrm{a}$, can lead the machine learning algorithm to fit the data with incorrect models, as shown by the blue line in $4 \mathrm{~b}$. In $4 \mathrm{c}$, artificial points added thanks to expert input eventually lead symbolic regression towards better models.

where $\tau_{c}$ is the numerical value of the flow stress for an arbitrary $\varepsilon^{p}$ value. Furthermore, $\hat{\dot{\varepsilon}}^{p}$ is added to the variables that symbolic regression can use, as it is apparent from previous tests that it is particularly useful to fit the data.

This strategy is equivalent to the process used to derive both MTS [32, 34-36] and PTW [16]. The two models are obtained from a modified Voce hardening law

$$
\frac{d \tau}{d \varepsilon^{p}}=\theta\left(\varepsilon^{p}, \dot{\varepsilon}^{p}, \mathrm{~T}\right)
$$

where $\theta$ is function of equivalent plastic strain, equivalent plastic strain rate and temperature. If $\tau$ is plotted against $\varepsilon^{p}$, as in Figures $3 \mathrm{c}$, the Voce hardening law is responsible for the shape of the curve. Moreover, the Voce law enforces saturation for high values of equivalent plastic strain.

Once $\theta$ is defined, the strength model is obtained by integration of $\theta$ with respect to $\varepsilon^{p}$ and an integration constant is introduced. Accordingly, integrating $f$ in Eq. (20) yields

$$
\tau=\tau_{c}+\int f\left(\varepsilon^{p}, \dot{\varepsilon}^{p}, \hat{\dot{\varepsilon}}^{p}, \mathrm{~T}\right) \mathrm{d} \varepsilon^{p},
$$

with $\tau_{c}$ denoting the integration constant and where $f$ is obtained through symbolic regression.

Saturation of $\tau$ is a highly desirable feature for most constitutive models. Classically, the Voce hardening law is derived from functions that saturate for large values of $\varepsilon^{p}$, such as $\tanh (x)$ or the Arrhenius form $e^{-1 / x}$ to mention a few. Within the symbolic regression approach, forcing the method to use a predefined form of the equation would result in a significant reduction of the solution space. In order to obtain a saturation value while using the largest possible solution space, the following condition is instead enforced

$$
\frac{d \tau}{d \varepsilon^{p}}=f\left(\varepsilon^{p}, \dot{\varepsilon}^{p}, \hat{\dot{\varepsilon}}^{p}, \mathrm{~T}\right) \geq 0
$$

The slope of $\tau$ in the $\varepsilon^{p}-\tau$ plane being always $\geq 0$ is clearly a less strict requirement than choosing a priori the form of $f$. The hardening law with the best compromise between fitting error and complexity, found through symbolic regression on the D1 to D5 datasets (see Appendix A), is

$$
\frac{d \tau}{d \varepsilon^{p}}=0.954 \min \left(\left[2074.4,\left(1248.204+1.129 \hat{\dot{\varepsilon}}^{p}-\mathrm{T}\right)^{\left(1.129-\varepsilon^{p}\right)}\right]\right)
$$

where, in order to avoid unexpected behavior for large values of $\varepsilon^{p}$, the following condition is also introduced

$$
\left.\left.\frac{d \tau}{d \varepsilon^{p}}\right|_{\varepsilon^{p}>1} \equiv \frac{d \tau}{d \varepsilon^{p}}\right|_{\varepsilon^{p}=1}
$$


Thus far, the expression for the integration constant $\tau_{c}$ in Eq. (23) is still unknown. Observing that for an arbitrary value of $\varepsilon^{p}$ a corresponding $\tau_{c}$ can be obtained with symbolic regression, two options for $\varepsilon^{p}$ appear reasonable. The first one is to compute the initial flow stress of the material by setting $\varepsilon^{p}=0$ and letting the symbolic regression determine $\tau_{c}\left(\varepsilon^{p}=0, \dot{\varepsilon}^{p}, \mathrm{~T}\right)$. The second choice is to compute the saturation flow stress with $\varepsilon^{p}=1$. Despite being reasonable, the former and latter strategy face experimental issues related to the Hopkinson bar compression test: the flow stress for $\varepsilon^{p}=0$ seems difficult to measure accurately due to initial oscillations, while the largest achievable value of equivalent plastic strain is limited by the kinetic energy of the striker bar which is not sufficient to produce plastic strain much larger than $20 \%$. Therefore, the value of $\varepsilon^{p}$ used is the smallest one at which one of the strain-stress curves terminates, and it is indicated with $\varepsilon_{f}^{p}$. For the dataset employed in the current work, $\varepsilon_{f}^{p}=0.23$. Interpolation is used to obtain $\left.\tau_{c}\right|_{\varepsilon^{p}=\varepsilon_{f}^{p}}$ for the dataset that don't have points for $\varepsilon^{p}=\varepsilon_{f}^{p}$.

The $\tau_{c}$ term is computed using the original dataset and the process used to generate it can be visualized as the path that takes from Figure 4a to Figure 4b. The complete expression is given by

$$
\tau_{c}=4.315 \cdot 10^{-10} \dot{\varepsilon}^{p}+149707.505 \max \left(\left[19.086, \hat{\dot{\varepsilon}}^{p}\right]\right)-183494.523-0.208 \mathrm{~T}-90.761 \operatorname{erfc}\left((0.004 \mathrm{~T})^{\hat{\dot{\varepsilon}}^{p}}\right),
$$

where erfc denotes the complementary error function. It is worth underlining that the term $\max \left(\left[19.086, \hat{\dot{\varepsilon}}^{p}\right]\right)$ introduces a discontinuity of the derivative at $\dot{\varepsilon}^{p} \approx 1.95 \cdot 10^{8}$ which captures the slope difference between the first (thermally activated dislocation motion) and second (phonon drag) parts of the training data Fig. 4a.

\section{4. $S R-4$}

The evident data gap depicted in Fig. 4a corresponds to the one observed in the experiments. During the learning process of SR-3, symbolic regression was expected to use a curve with positive slope to interpolate over the data gap. The SR-3 $\tau_{c}$ curve, Eq. (27), exhibits instead a plateau for strain rates from $10^{5}$ to $10^{8}$, similar to the one depicted in Figure 4b. Therefore, additional artificial points have been added to fill the gap and, lacking better knowledge, a line has been used to connect the last experimental point for low strain rates to the first one for high strain rates, see Fig. 4c. Drawing a line in the log-log space implies assuming a power law for intermediate strain-rates as in [16]. Finally, the chosen form of $\tau_{c}$ is

$$
\tau_{c}=528.501+0.245 \hat{\dot{\varepsilon}}^{p}-0.170 \mathrm{~T}+\left(0.415 \hat{\dot{\varepsilon}}^{p}\left(\dot{\varepsilon}^{p}\right)^{0.151}-39.845\right) \hat{\mathrm{T}}
$$

For the sake of clarity, the four models obtained through symbolic regression are summarized below

- SR-1 indicates the model described by Eq. (17), it is obtained using the datasets D1, D2 and D3 in Appendix A

- SR-2 denotes the strength model derived using the datasets D1 to D5 in Appendix A and expert knowledge to enforce an asymptotic behavior for large strains. It is defined by Eq. (18),

- The learning process of SR-3 is carried out in two phases. The slope of the curve is given by Eq. (25) and its value, $\tau_{c}$, for $\varepsilon^{p}=\varepsilon_{f}^{p}$ is expressed in Eq. (27). The datasets are D1 to D5 in Appendix A,

- SR-4 uses the same equation of SR-3 for the slope, Eq. (25), whereas Eq. (28) is employed to compute $\tau_{c}$. The training dataset is the same used for SR-3, enriched with artificial points to fill the gap for intermediate strain rates, see Fig. 4c and Appendix A.

Finally, it is worth remarking that all the numerical coefficients appearing in the equations defining the four strength models are kept constant during the verification carried out in Section 6. The numerical coefficients for MTS and PTW are computed as described in [32] and [16], respectively. Calibration of MTS and PTW parameters can be seen as a classical regression problem: given an equation with unknown coefficients find the coefficients that minimize the distance between the curve and the experimental data. Conversely, symbolic regression finds the functional form, and the coefficients, that best "fit" the experimental data. Calibration of MTS and PTW can be solved in polynomial time whereas symbolic regression seeks a solution of a non-polynomial problem. 


\section{Computational formulation}

Hereafter, the computational framework and numerical manipulations necessary to implement the strength models in Sections 3 and 4 as an ABAQUS [38] user material (VUMAT) are described. In this work, the constitutive model is formulated in a corotational hypoelastic framework. The Green-Naghdi stress rate is used to neutralize rotations in the presence of finite deformations and standard radial return algorithms are employed [33, 39]. From a converged state at time $t^{(n)}$ the time is advanced to $t^{(n+1)}=t^{(n)}+\Delta t$. Quantities from the previous converged state, indicated with $\bullet^{(n)}$, and the total strain increment $\Delta \varepsilon$ are given at the beginning of the step and the updated stress and history variables are computed for $t^{(n+1)}$. Accordingly with the corotational framework, the total strain increment is obtained through an objective rate of deformation tensor and the elastic strains are assumed to be small (which is usually true for metals). It is also worth underlining that the strength models used in this work can be employed, without modification, within a hyperelastic framework [40].

Decomposition in volumetric part, $\overline{\boldsymbol{}}=\frac{1}{3} \operatorname{trace}(\bullet)$, and deviatoric component, $\overline{\boldsymbol{\bullet}}$, of the strain tensor

$$
\boldsymbol{\varepsilon}=\bar{\varepsilon} \mathbf{1}+\tilde{\varepsilon},
$$

and stress tensor

$$
\sigma=\bar{\sigma} \mathbf{1}+\tilde{\boldsymbol{\sigma}},
$$

is used where the rank two identity tensor is represented by $\mathbf{1}$.

\subsection{Volumetric: equation of state}

The pressure component $p=-\bar{\sigma}$, computed using a constant bulk modulus, does not take into account shocks inside the material due to high-impact velocities. Therefore, pressure at the Gauss integration point is obtained from a Mie-Grüneisen type equation of state. Using a linear Hugoniot shock velocity [41] yields

$$
p=\rho_{0} c_{0}^{2} \frac{1+\left(1-\frac{1}{2} \Gamma_{0}\right) \mu-\frac{1}{2} b \mu^{2}}{(1-(s-1) \mu)^{2}} \mu+\left(\Gamma_{0}+b \mu\right) E,
$$

for $\mu>0$, or

$$
p=\mu \rho_{0} c_{0}^{2}+\Gamma_{0} E
$$

if $\mu \leq 0$, with the measure of compression, $\mu$, given by

$$
\mu=\frac{\rho}{\rho_{0}}-1 \approx-3 \bar{\varepsilon}
$$

and where $c_{0}, \rho_{0}, \rho$, and $E$ are the initial sound speed, the initial density, the density at the current time and the internal energy-per-unit reference volume, respectively. Due to $\mathrm{J}_{2}$ plasticity, the increment of volumetric strain is entirely elastic: hence $\mu^{(n+1)}=\mu^{(n)}+\Delta \mu \approx \mu^{(n)}-3 \Delta \bar{\varepsilon}$. Although $\mu^{(n)}$ could be derived from $\bar{\sigma}^{(n)}$, the converged value of $\mu$ is stored, for convenience, as a history variable.

The constants $\Gamma_{0}=1.96$ and $s=1.5$, are used in Eqs. (31)-(32). The value of the initial bulk modulus is

$$
K_{0}=2 G_{0} \frac{1+v}{3(1-2 v)},
$$

with $G_{0}=46.269 \mathrm{GPa}$ and $v=0.34$. Finally, the initial sound speed is obtained from $c_{0}=\sqrt{K_{0} / \rho_{0}}$ where $\rho_{0}$ is given, for each test case, in Section 6. 


\subsection{Deviatoric: $J_{2}$ plasticity}

The total deviatoric strain rate is additively decomposed into elastic and plastic contributions, which in incremental form can be written as

$$
\Delta \tilde{\boldsymbol{\varepsilon}}=\Delta \tilde{\boldsymbol{\varepsilon}}^{e}+\Delta \tilde{\boldsymbol{\varepsilon}}^{p}
$$

where the $e$ and $p$ superscripts indicate the elastic and plastic part, respectively. The classical Von Mises yield surface is considered

$$
f_{\lambda}:=\|\boldsymbol{s}\|-\sqrt{\frac{2}{3}} \tau \leq 0,
$$

and the flow stress, $\tau$, is obtained from the strength models described in Sections 3 and 4. An associative flow rule is employed, using the customary radial return algorithm leads to

$$
\Delta \tilde{\boldsymbol{\varepsilon}}^{p}=\boldsymbol{n} \Delta \lambda
$$

with

$$
\boldsymbol{n}=\frac{\tilde{\boldsymbol{\sigma}}}{\|\tilde{\boldsymbol{\sigma}}\|},
$$

where the plastic multiplier $\Delta \lambda \geq 0$ is computed as in [39]. The equivalent plastic strain is obtained from the integral over time of the equivalent plastic strain rate

$$
\varepsilon^{p}=\int \dot{\varepsilon}^{p} \mathrm{dt}
$$

or, incrementally,

$$
\varepsilon^{p^{(n+1)}}=\varepsilon^{p^{(n)}}+\Delta \varepsilon^{p}
$$

where

$$
\Delta \varepsilon^{p}=\sqrt{\frac{2}{3}} \Delta \lambda
$$

with the equivalent plastic strain rate given by $\dot{\varepsilon}^{p}=\frac{\Delta \varepsilon^{p}}{\Delta t}$. Increase in temperature, $\Delta \mathrm{T}$, is due to plastic dissipation

$$
\Delta \mathrm{T}=\Psi \frac{\tilde{\sigma}: \Delta \tilde{\varepsilon}^{p}}{\rho C_{p}} .
$$

where $\Psi$ is the Taylor-Quinney coefficient and $C_{p}$ is the specific heat capacity of the material. Time integration is carried out as in [33] and a Newton-Raphson iterative scheme is used to compute the converged values for $\tilde{\sigma}, \varepsilon^{p}, \dot{\varepsilon}^{p}$ and $\mathrm{T}$ at time $t^{(n+1)}$.

The remainder of the present section is concerned with the treatment of singularities or undesirable behavior of the considered strength models. Most of the singularities involve the $\ln \left(\varepsilon^{p}\right)$ and $\ln \left(\dot{\varepsilon}^{p}\right)$ terms that, similarly to the method described in [42], are modified by adding a small perturbation

$$
\ln \left(\varepsilon^{p}\right) \rightarrow \ln \left(\varepsilon^{p}+\delta\right), \quad \ln \left(\dot{\varepsilon}^{p}\right) \rightarrow \ln \left(\dot{\varepsilon}^{p}+\dot{\delta}\right) .
$$

Higher values of $\delta$ (or $\dot{\delta}$ ) lead to faster convergence (i.e. fewer Newton-Raphson iterations) whereas smaller values cause slower convergence. The perturbation terms $\delta$ and $\dot{\delta}$ vary for each strength model, and their value is set to obtain a reasonable compromise between speed and accuracy. Clearly, for low strain rates and very long simulation times, this approach would be ill-suited: but it produces good results for the application of interest (Section 6). 


\subsubsection{MTS and PTW}

Implementation of MTS follows [33]. The only difference is in the perturbations of $\varepsilon^{p}$ and $\dot{\varepsilon}^{p}$ equal to $\delta^{M T S}=0$ and $\dot{\delta}^{M T S}=1.0 \cdot 10^{-8} \dot{\varepsilon}_{\max }^{p}$ where $\dot{\varepsilon}_{\max }^{p}$ is a MTS parameter. When the PTW model is considered, a corner case has to be handled: if the saturation and yield stress are identical $\left(\bar{\tau}_{s} \equiv \bar{\tau}_{y}\right)$ or if $s_{0}=\bar{\tau}_{y}$, the flow stress becomes $\bar{\tau}=\bar{\tau}_{s}$. For PTW, $\delta^{P T W}=0$ and $\dot{\delta}^{P T W}=1.0 \cdot 10^{-10} \dot{\xi}$.

\subsection{2. $S R-1$ and $S R-2$}

For the first and second models generated by symbolic regression, both $\varepsilon^{p}$ and $\dot{\varepsilon}^{p}$ are perturbed. The perturbations of the equivalent plastic strain rates are $\dot{\delta}^{S R-1}=1.0 \cdot 10^{-12}$ and $\dot{\delta}^{S R-2}=3.0 \cdot 10^{-12}$. For high values of equivalent plastic strain, the $\tau-\varepsilon^{p}$ curve has negative slope (see Fig. 3b). Therefore, $\varepsilon^{p}$ is modified as follows

$$
\varepsilon^{p^{S R-1}}=\min \left(\left[0.2, \varepsilon^{p}+1.0 \cdot 10^{-10}\right]\right), \quad \quad \varepsilon^{p^{S R-2}}=\min \left(\left[0.4, \varepsilon^{p}+3.0 \cdot 10^{-12}\right]\right) .
$$

Considering a value of equivalent plastic strain, set as the minimum between the current $\varepsilon^{p}$ and 0.2 for SR-1, or 0.4 for SR-2, enforces a saturation value of the $\tau-\varepsilon^{p}$ curve. It is worth remarking that despite trying to force the saturation behavior of SR-2 through additional data, Fig. 3c, an additional condition has to be introduced.

\subsection{3. $S R-3$ and $S R-4$}

The third and fourth model, SR-3 and SR-4, are generated by requiring that the $\tau-\varepsilon^{p}$ curve possesses a positive slope. Nonetheless, the conditions

$$
\varepsilon^{p^{S R-3}}=\min \left(\left[1.0, \varepsilon^{p}\right]\right), \quad \quad \varepsilon^{p^{S R-4}}=\min \left(\left[1.0, \varepsilon^{p}\right]\right),
$$

are introduced to avoid extrapolation: the largest value of the equivalent plastic strain in the training dataset is $\varepsilon^{p}=1$ and for this large value of $\varepsilon^{p}$ the $\tau-\varepsilon^{p}$ curve is already saturated. The values of the perturbations used for the two models are $\delta^{S R-3}=\delta^{S R-4}=0$ and $\dot{\delta}^{S R-3}=\dot{\delta}^{S R-4}=1.0 \cdot 10^{-6}$.

\section{Verification}

Assessment of the strength models obtained through symbolic regression is carried out by means of a classical large-deformation and high strain-rate test. Classically, machine learning validation is performed by partitioning the experimental data into training and validation sets (unseen data). In our case, the number of stress-strain curves obtained from experiments is very limited, typically one curve (or only one point) for a given strain rate and temperature. Therefore, all the available experimental stress-strain curves have been used to train the symbolic regression. Validation on unseen data is hence carried out by resorting to a thorough test case, the Taylor anvil impact [15], where all the Gauss integration points are exposed to a wide range of strain rates and temperatures, allowing to evaluate almost the entire space represented by $\tau$.

A copper cylinder is moving with an initial velocity $\mathrm{U}_{0}$ towards a rigid anvil. At the beginning of the analysis all the energy is stored as kinetic energy that, by the end of the analysis, is completely dissipated by the plastic deformation of the cylinder. In the current work two categories of experimental data are used to validate the strength models: the deformed profile of the recovered sample, if available, and the final length of the deformed cylinder, L. The profiles and final lengths obtained from the symbolic regression models are also compared to the state-of-the art models MTS and PTW (the plastic strain rates lie in a range where MTS is still physically applicable). Time integration is performed explicitly and axisymmetric four-node elements (CAX4R) are used. Moreover, friction between cylinder head and anvil is introduced using a friction coefficient equal to 0.1 [33].

The first set of test cases [41] involves two cylinders with initial lengths, $\mathrm{L}_{0}$, and velocities equal to $25.4 \mathrm{~mm}$ and $146 \mathrm{~m} / \mathrm{s}$ and $50.8 \mathrm{~mm}$ and $177 \mathrm{~m} / \mathrm{s}$, respectively. The initial diameter of the samples is $7.72 \mathrm{~mm}$, the initial temperature is $298 \mathrm{~K}$ and the initial density is $8900 \mathrm{Kg} / \mathrm{m}^{3}$. Adiabatic conditions are assumed throughout the simulation and the Taylor-Quinney coefficient is taken to be $\Psi=0.95$ [33]. The percent errors on L, obtained from

$$
\text { 100. }\left(1-\frac{L^{\text {model }}}{L^{\text {ref }}}\right) \text {, }
$$


for a given strength model are listed in Table. 1 and the computed deformed profiles are compared to the experimental, $L^{r e f}$, ones in Figs. 5 and 6. MTS and PTW are in good agreement with the experimental results and, to a lesser degree, also the model denoted with SR-4. SR-2 and SR-3 are performing well for the test with initial velocity equal to 146 $\mathrm{m} / \mathrm{s}$ whereas they predicted deformed profile for the second test is quite off. It should be remarked that, due to the chosen axis scaling, the error on the radial direction is magnified in the plots. Clearly, model SR-1 does not perform well and it is therefore discarded in the subsequent comparisons.

In order to validate the models it is also useful to look at some internal variables, accumulated equivalent plastic strain and temperature in this case, in the deformed configuration. Contour plots of the accumulated equivalent plastic strain and temperature at the final time are shown in Figs 7 and 8 for the cylinder with initial velocity equal to 146 $\mathrm{m} / \mathrm{s}$. Temperature and accumulated plastic strain are clearly higher in the proximity of the contact surface where most of the kinetic energy is dissipated. Model SR-4 predicts a temperature field very similar to that obtained from PTW whereas the equivalent plastic strain values are between the ones predicted by MTS and PTW. Although SR-2 and SR-3 over-predict the maximum value of equivalent plastic strain, the temperature field seems to be in reasonable agreement with PTW and MTS.

Table 1: Percent errors on L for the tests in Figs. 5 and 6

\begin{tabular}{|cccrrrrrr|}
\hline Initial velocity $[\mathrm{m} / \mathrm{s}]$ & Temperature $[\mathrm{K}]$ & $L^{\text {ref }}[\mathrm{mm}]$ & MTS & PTW & SR-1 & SR-2 & SR-3 & SR-4 \\
\hline 146.0 & 298 & 18.77 & -1.14 & -0.48 & 1.72 & 0.65 & -0.44 & -2.17 \\
177.0 & 298 & 33.62 & 0.79 & 1.01 & 5.51 & 3.94 & 1.68 & -0.93 \\
\hline
\end{tabular}

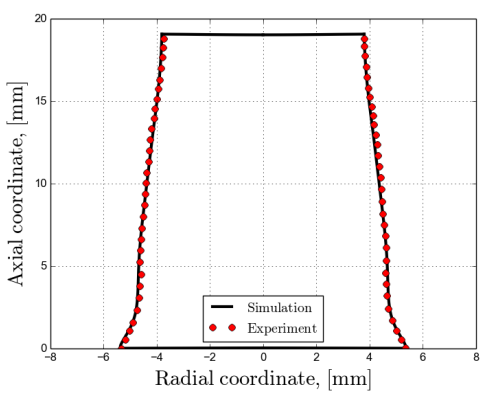

(a) MTS

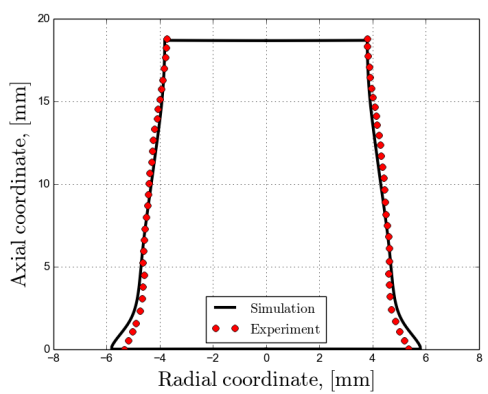

(d) SR-2

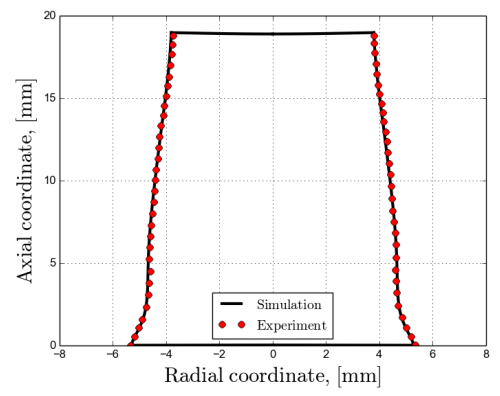

(b) PTW

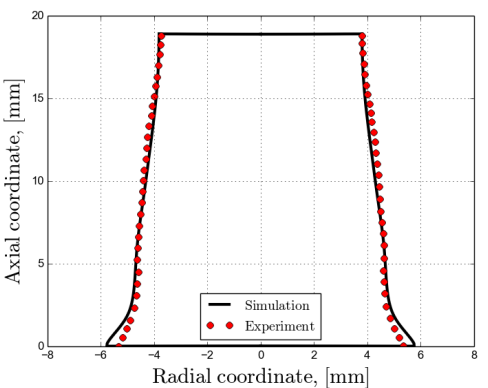

(e) SR-3

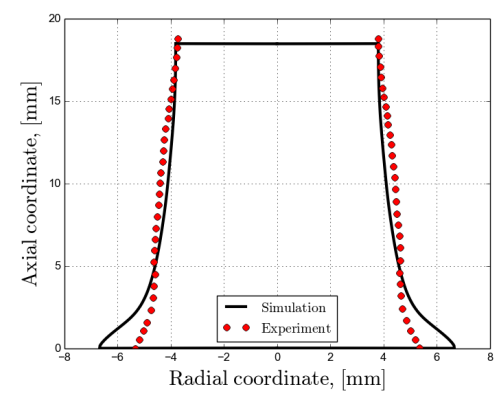

(c) SR-1

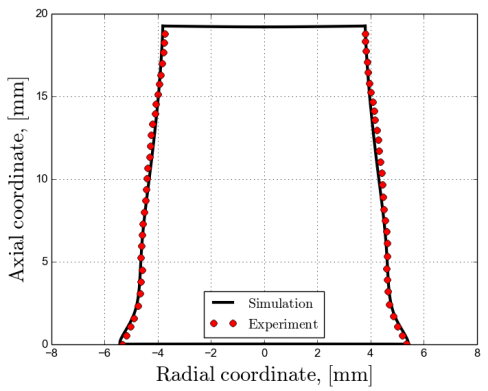

(f) SR-4

Figure 5: Computed profiles vs. experimental results, initial velocity $146 \mathrm{~m} / \mathrm{s}$ at room temperature: a) standard MTS model, b) standard PTW model, c) simplest symbolic regression model, no expert knowledge, d) strength model obtained from stress-strain data with additional expert knowledge,

e) Voce hardening expression from symbolic regression, f) Voce hardening from symbolic regression and expert knowledge on saturation stress

During the second part of the validation process all the copper Taylor anvil impacts presented in [43] are simulated. The initial length and diameter for all the cylinders are equal to $30 \mathrm{~mm}$ and $6 \mathrm{~mm}$ respectively. Initial velocities range between $48.7 \mathrm{~m} / \mathrm{s}$ and $394 \mathrm{~m} / \mathrm{s}$ whereas initial temperatures are comprised within $295 \mathrm{~K}$ and $1235 \mathrm{~K}$. Initial temperature and velocity for all the 37 shots are listed in Table 2. Furthermore, although copper's density is approximately $8900 \mathrm{Kg} / \mathrm{m}^{3}$, the exact values given in [43] are used for each test. 


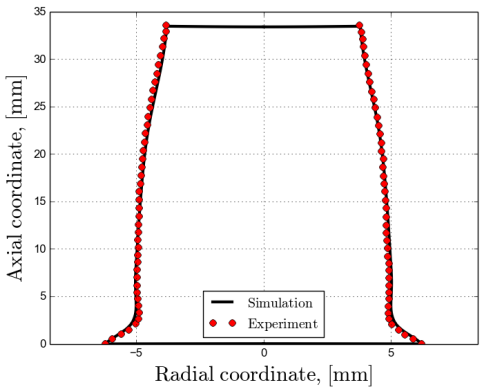

(a) MTS

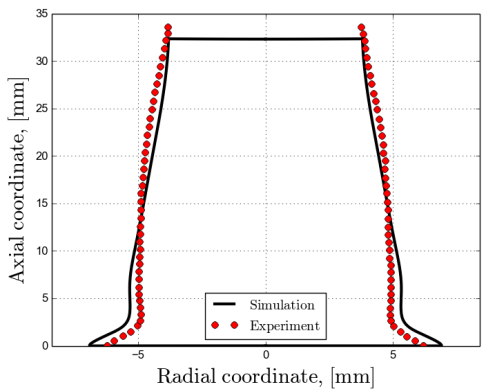

(d) SR-2

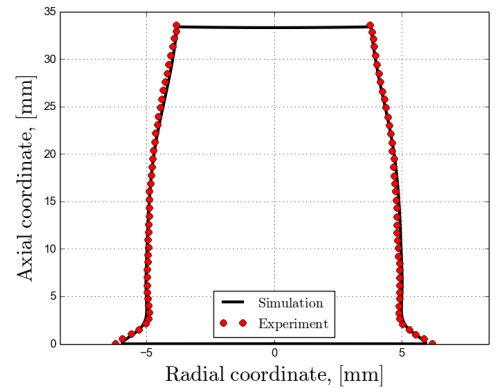

(b) PTW

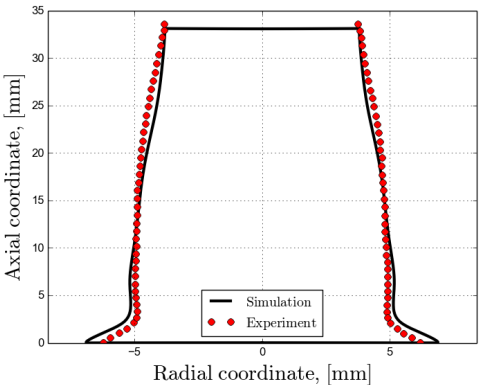

(e) SR-3

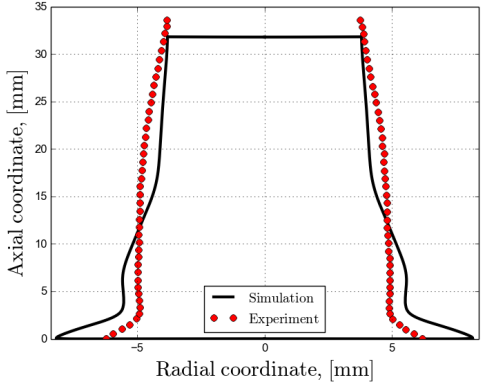

(c) SR-1

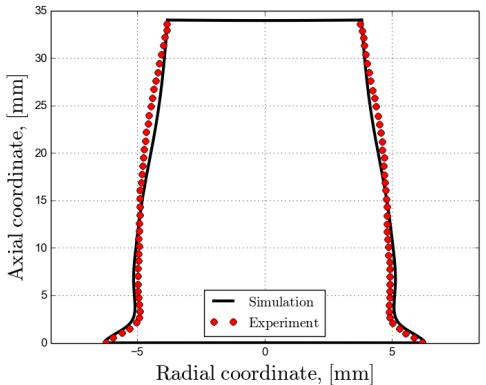

(f) SR-4

Figure 6: Computed profiles vs. experimental results, initial velocity $177 \mathrm{~m} / \mathrm{s}$ at room temperature: a) standard MTS model, b) standard PTW model, c) simplest symbolic regression model, no expert knowledge, d) strength model obtained from stress-strain data with additional expert knowledge, e) Voce hardening expression from symbolic regression, f) Voce hardening from symbolic regression and expert knowledge on saturation stress

Depending on the initial temperature of the projectile, the tests are collected within three groups: (i) room temperature shots with $\mathrm{T}=295 \mathrm{~K}$, (ii) shots with temperature varying in the [700, 780] $\mathrm{K}$ interval and (iii) shots with $T \geq 1230 \mathrm{~K}$. An average percent error for each model and temperature group is then computed according to

$$
\frac{1}{N} \sum_{i}^{N}\left|\mathrm{e}_{i}^{\text {model }}\right|,
$$

where $N$ is the numerosity of the group and $\mathrm{e}_{i}^{\text {model }}$ represent the percent error on $\mathrm{L} / \mathrm{L}_{0}$ for $i$-th test and the employed model. The average errors for the first two groups are computed discarding shots 23, 34, 63 and 53 . Test 23 is discarded because although it is almost identical to the one whose results are shown in Fig. 5 the percent error is much larger than the error in Table 1 for all strength models. Shot 63 has been discarded because while all the models seem to agree on the final length of the deformed cylinder the experimental initial speed is uncertain (see note in [43]). Shots 34 and 53 are discarded because all the models have an absolute value of the percent error larger than 10\%: this is an indicator that physical phenomena, other then plasticity, are happening. Due to the high initial speed of test 53, on the recovered sample "large peripheral cracks" are observed. Therefore, damage should be included in the material model to correctly represent the cylinder's response. Finally, test 34 is performed at high initial velocity and temperature. During the plastic deformation process the computed temperature in the sample increases and approaches the melting temperature $1357 \mathrm{~K}$ of copper. Material's melting temperature is a function of the applied pressure [44] (or volumetric strain [29]) but such a relationship is not accounted for in the present work. Neglecting the relation between melting temperature and pressure is thought to lead to the poor results of the experiments in group (iii).

From Table 2 it is evident that MTS, PTW and SR-4 models have similar performance and are accurate in computing the final length of the deformed sample for temperature groups (i) and (ii). SR-2 and SR-3 perform reasonably well at lower strain rates but start to be inaccurate for high initial velocities, $U_{0}>200 \mathrm{~m} / \mathrm{s}$. The reason of this inaccuracy is that the stress-strain data used to develop the models have a gap between $\dot{\varepsilon}^{p}>1 \cdot 10^{5}$ and $\dot{\varepsilon}^{p}<1 \cdot 10^{8}$. Final profile of the recovered sample is compared to the numerical simulations (MTS, PTW and SR-4 only) for shots 

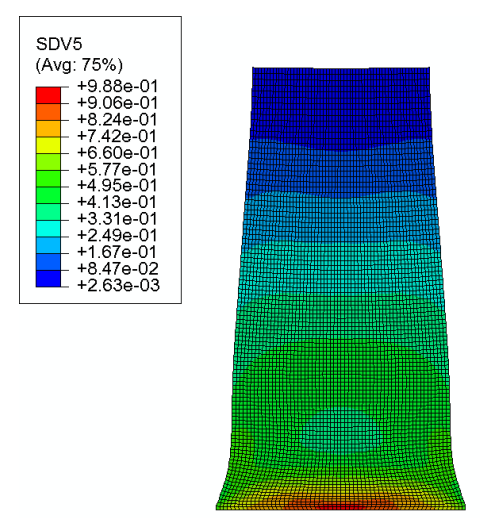

(a) MTS
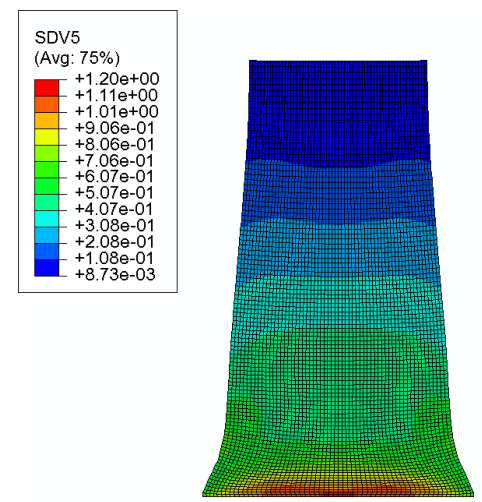

(d) SR-2
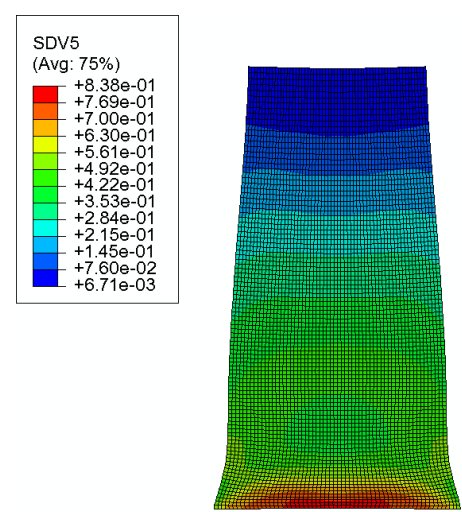

(b) PTW
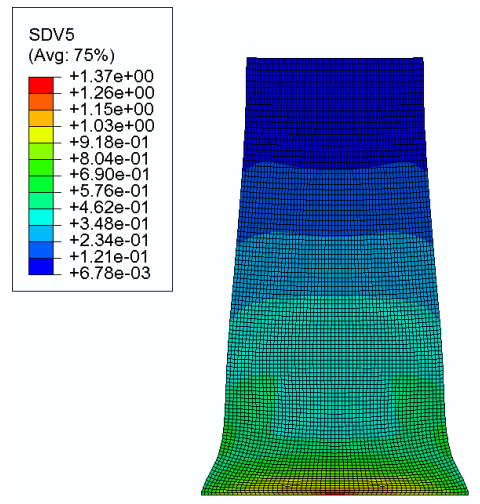

(e) SR-3
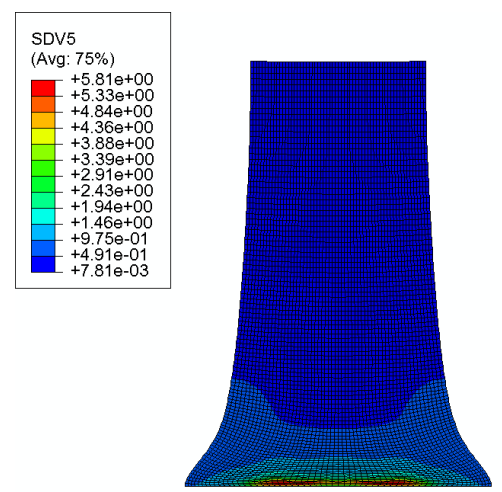

(c) SR-1
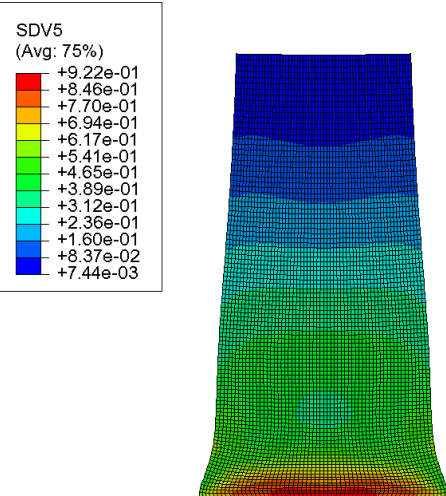

(f) SR-4

Figure 7: Equivalent plastic strain, initial velocity $146 \mathrm{~m} / \mathrm{s}$ at room temperature: a) standard MTS model, b) standard PTW model, c) simplest symbolic regression model, no expert knowledge, d) strength model obtained from stress-strain data with additional expert knowledge, e) Voce hardening expression from symbolic regression, f) Voce hardening from symbolic regression and expert knowledge on saturation stress

26, 52, and 61 in Fig. 9, Fig. 10 and Fig. 11, respectively. The numerical results obtained with the different strength models are in good agreement with each other and close to the experimental profiles.

Finally, contour plots of temperature and accumulated plastic strain are shown for test 61 in Fig. 12 and Fig. 13. Temperatures and plastic strains computed by MTS, PTW and SR-4 in the final state are remarkably similar (difference in $\varepsilon^{p} \approx 3 \%$ ). It should be underlined that due to the numerical treatment of the terms $\ln \left(\varepsilon^{p}\right)$ and $\ln \left(\dot{\varepsilon}^{p}\right)$ appearing in the strength models (see Section 5) the minimum accumulated plastic strain is not zero, as it would be expected. Nonetheless, the value is three orders of magnitude smaller than the maximum $\varepsilon^{p}$ which makes the adopted solution adequate for the case in study.

For experiments performed at very high temperatures, group (iii), all the strength models fail, see Table 2 . Nonetheless, it is quite interesting to observe the results of the SR-3 model; it seems to outperform all the other models and one might conclude that the model is capable of representing the material behavior at high temperatures. This result is fortuitous since no experimental data has been fed to the symbolic regression tool to justify this behavior. SR-3 is an extremely good example of how data driven models can be misleading. Although this point will be touched on more later, expert knowledge is still a vital component of model development.

Lastly, two remarks. i) For several tests at room temperature two initial velocities are given in [43]: one measures optically, $\mathrm{U}_{0}^{o p t}$, and one from pin reading, $\mathrm{U}_{0}^{\text {pin }}$. The maximum difference in the two values of initial velocity can be as high as $7 \%$. Therefore, when two values for $\mathrm{U}_{0}$ are given, the used value is the one belonging to the vector $\left\{\min \left(\left[\mathrm{U}_{0}^{o p t}, \mathrm{U}_{0}^{p i n}\right]\right), \max \left(\left[\mathrm{U}_{0}^{o p t}, \mathrm{U}_{0}^{\text {pin }}\right]\right)\right.$, mean $\left.\left(\left[\mathrm{U}_{0}^{\text {opt }}, \mathrm{U}_{0}^{\text {pin }}\right]\right)\right\}$ that givens the lowest average percent error on $\mathrm{L}$ for MTS, PTW and SR-4. ii) Numerical solutions that have a $2-3 \%$ average error on $\mathrm{L}$ are considered accurate since the 

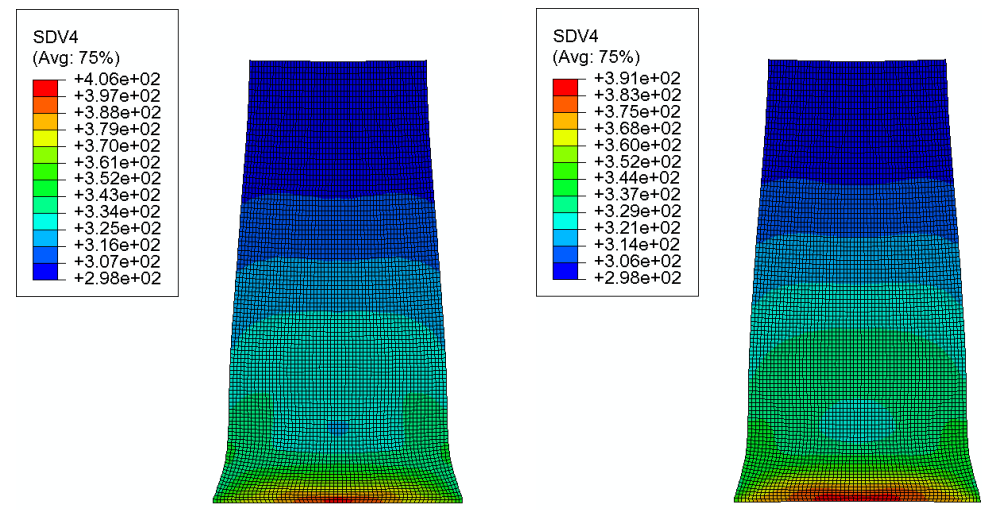

(b) PTW

(a) MTS

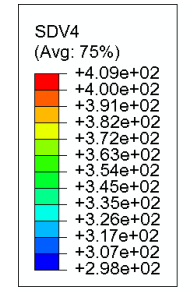

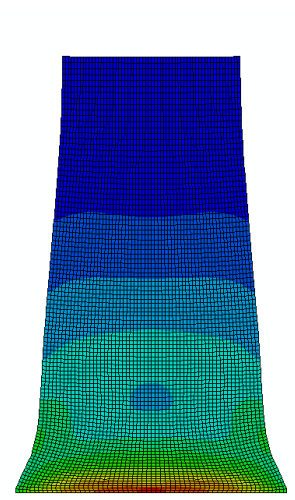

(d) SR-2
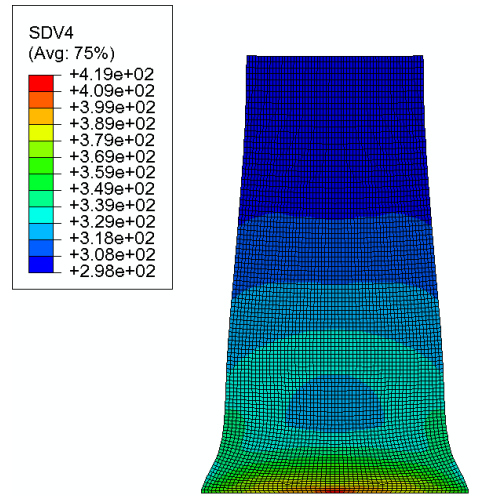

(e) SR-3
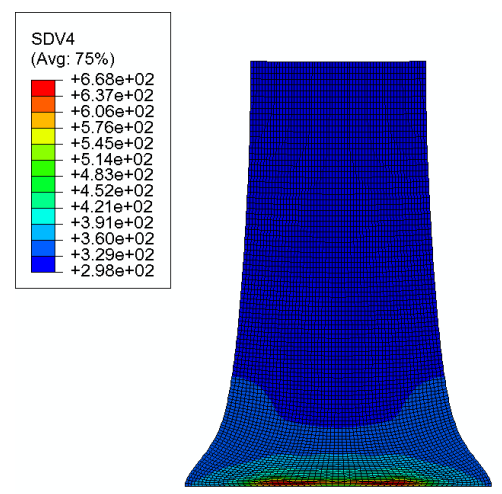

(c) SR-1

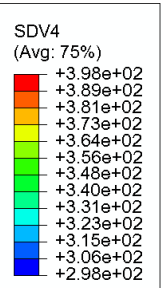

(f) SR-4

Figure 8: Temperature, initial velocity $146 \mathrm{~m} / \mathrm{s}$ at room temperature: a) standard MTS model, b) standard PTW model, c) simplest symbolic regression model, no expert knowledge, d) strength model obtained from stress-strain data with additional expert knowledge, e) Voce hardening expression from symbolic regression, f) Voce hardening from symbolic regression and expert knowledge on saturation stress

experimental uncertainty has to be accounted for. Initial velocity and temperature for tests 56 and 61 in Table 2 are practically identical and the difference in final length is $\approx 2 \%$. Although a $2 \%$ error is exceptionally good from the experimental point of view, it reduces the confidence of the results obtained in the validation process. 


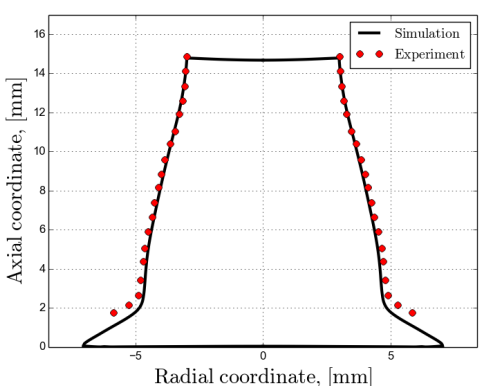

(a) MTS

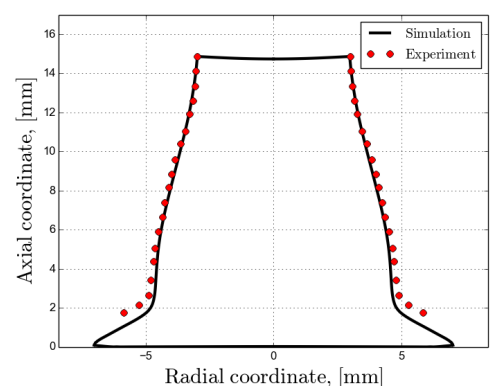

(b) PTW

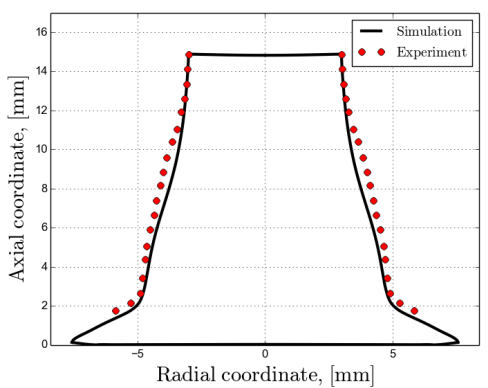

(c) SR-4

Figure 9: Computed profiles vs. experimental results for shot \# 26: a) standard MTS model, b) standard PTW model, c) Voce hardening from symbolic regression and expert knowledge on saturation stress

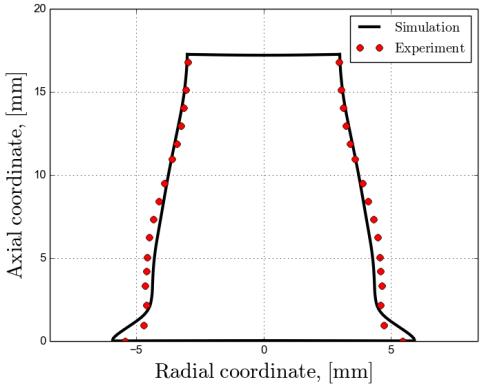

(a) MTS

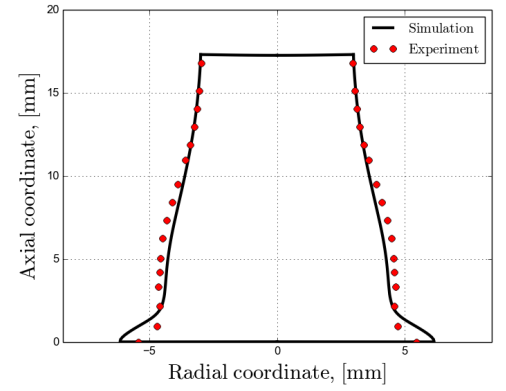

(b) PTW

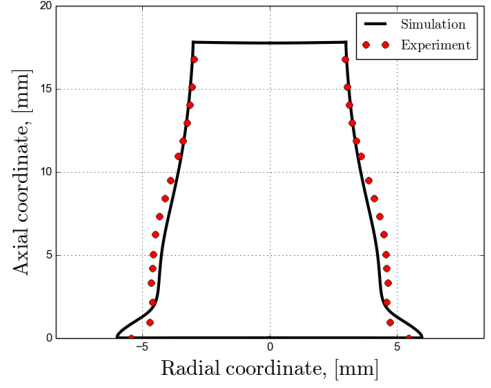

(c) SR-4

Figure 10: Computed profiles vs. experimental results for shot \# 52.

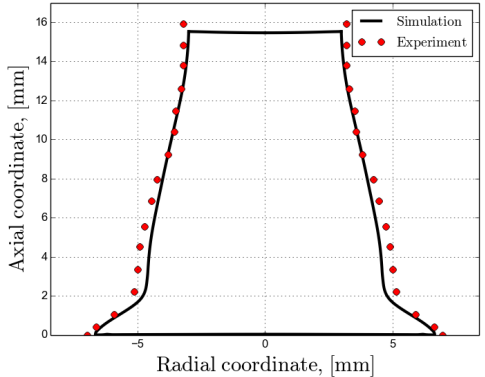

(a) MTS

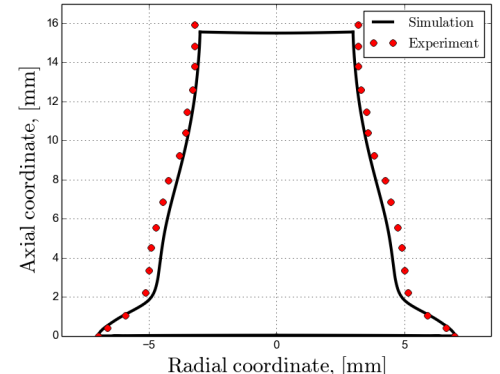

(b) PTW

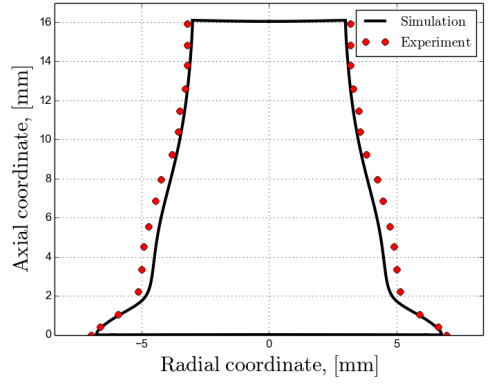

(c) SR-4

Figure 11: Computed profiles vs. experimental results for shot \# 61: a) standard MTS model, b) standard PTW model, c) Voce hardening from symbolic regression and expert knowledge on saturation stress 
Table 2: Errors on L for the 37 shots in [43]; error averages are computed discarding the shots marked with *

\begin{tabular}{|c|c|c|c|c|c|c|c|}
\hline Shot \# & Initial velocity $[\mathrm{m} / \mathrm{s}]$ & Temperature [K] & MTS & PTW & SR-2 & SR-3 & SR-4 \\
\hline 32 & 109.0 & 295 & 0.50 & 0.88 & 1.36 & 0.78 & -0.55 \\
\hline 33 & 126.0 & 295 & 0.45 & 0.82 & 1.53 & 0.75 & -0.84 \\
\hline 109 & 138.0 & 295 & -2.58 & -2.29 & -1.22 & -2.17 & -4.07 \\
\hline$* 23$ & 148.0 & 295 & 9.60 & 9.77 & 11.08 & 10.14 & 8.21 \\
\hline 108 & 165.0 & 295 & 1.49 & 1.59 & 3.83 & 2.57 & -0.14 \\
\hline 110 & 180.0 & 295 & 0.50 & 0.62 & 3.50 & 2.04 & -1.31 \\
\hline 24 & 181.0 & 295 & 2.03 & 2.14 & 5.02 & 3.57 & 0.22 \\
\hline 111 & 213.0 & 295 & 0.58 & 0.39 & 5.06 & 3.41 & -0.94 \\
\hline 31 & 219.0 & 295 & 2.02 & 1.92 & 6.81 & 5.25 & 0.62 \\
\hline 26 & 267.5 & 295 & 1.56 & 1.06 & 9.45 & 8.42 & 0.52 \\
\hline 25 & 307.0 & 295 & 1.68 & 1.00 & 9.96 & 1.34 & 1.36 \\
\hline 30 & 321.0 & 295 & -1.54 & -1.79 & 11.78 & -17.96 & -1.09 \\
\hline 29 & 332.0 & 295 & -4.79 & -5.03 & 8.88 & 8.97 & -4.28 \\
\hline 27 & 348.5 & 295 & -2.11 & -2.44 & -21.32 & -42.28 & -0.76 \\
\hline 35 & 349.0 & 295 & -1.88 & -2.01 & -6.18 & -30.15 & -0.53 \\
\hline 28 & 354.0 & 295 & -1.46 & -1.79 & -18.34 & -53.71 & 0.05 \\
\hline$* 34$ & 394.0 & 295 & -10.68 & -27.80 & -76.09 & -93.22 & -48.45 \\
\hline$* 63$ & 48.7 & 718 & 8.22 & 8.54 & 8.54 & 8.29 & 8.04 \\
\hline 62 & 115.0 & 721 & 2.07 & 1.77 & 1.41 & 2.71 & 0.31 \\
\hline 76 & 138.0 & 713 & -1.45 & -1.91 & -2.21 & -0.29 & -3.77 \\
\hline 60 & 142.0 & 717 & 4.35 & 3.88 & 3.56 & 5.57 & 2.05 \\
\hline 55 & 145.0 & 703 & 1.23 & 0.73 & 0.49 & 2.50 & -1.14 \\
\hline 74 & 185.0 & 733 & -0.46 & -0.83 & -2.17 & 2.72 & -3.84 \\
\hline 52 & 188.0 & 718 & -2.48 & -2.86 & -3.88 & 0.94 & -5.85 \\
\hline 58 & 190.0 & 733 & 1.67 & 1.35 & 0.01 & 5.19 & -1.73 \\
\hline 64 & 191.0 & 725 & -0.27 & -0.59 & -1.84 & 3.36 & -3.68 \\
\hline 57 & 206.0 & 724 & -1.59 & -1.92 & -3.42 & 3.18 & -5.42 \\
\hline 75 & 208.0 & 743 & 2.34 & 2.20 & 0.18 & 7.29 & -1.60 \\
\hline 56 & 210.0 & 721 & -2.23 & -2.45 & -4.04 & 2.90 & -6.16 \\
\hline 61 & 211.0 & 727 & 3.06 & 2.85 & 1.15 & 8.14 & -0.72 \\
\hline$* 53$ & 237.0 & 765 & -13.73 & -12.83 & -17.77 & -3.72 & -18.97 \\
\hline 81 & 127.0 & 1235 & -19.50 & -17.87 & -31.31 & -15.44 & -29.48 \\
\hline 77 & 135.0 & 1230 & -9.84 & -8.18 & -21.96 & -5.04 & -19.75 \\
\hline 82 & 155.0 & 1235 & -15.53 & -12.19 & -32.60 & -5.83 & -28.24 \\
\hline 79 & 157.0 & 1235 & -18.26 & -14.61 & -36.14 & -7.74 & -31.57 \\
\hline 83 & 178.0 & 1235 & -29.46 & -23.31 & -56.46 & -11.79 & -47.18 \\
\hline 78 & 181.0 & 1235 & -20.33 & -14.22 & -46.22 & -2.88 & -37.17 \\
\hline \multicolumn{3}{|c|}{ Average errors, group (i) } & 1.68 & 1.72 & 7.62 & 12.22 & 1.15 \\
\hline \multicolumn{3}{|c|}{ Average errors, group (ii) } & 1.93 & 1.95 & 2.03 & 3.73 & 3.02 \\
\hline \multicolumn{3}{|c|}{ Average errors, group (iii) } & 18.82 & 15.06 & 37.45 & 8.12 & 32.23 \\
\hline
\end{tabular}




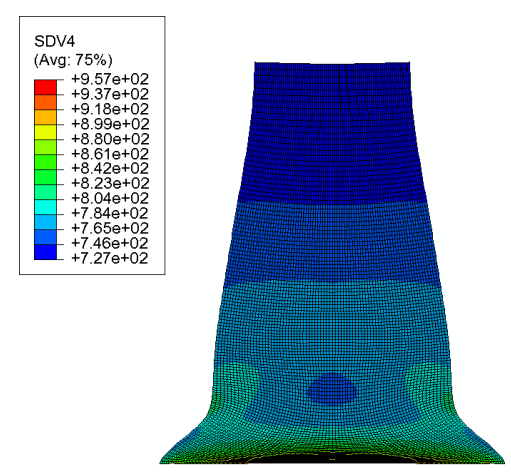

(a) MTS, temperature [K]

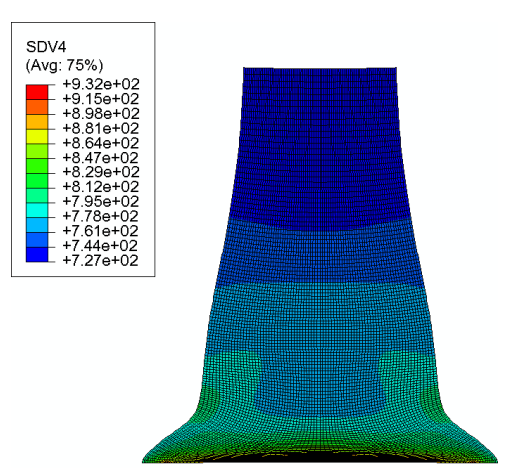

(b) PTW, temperature [K]

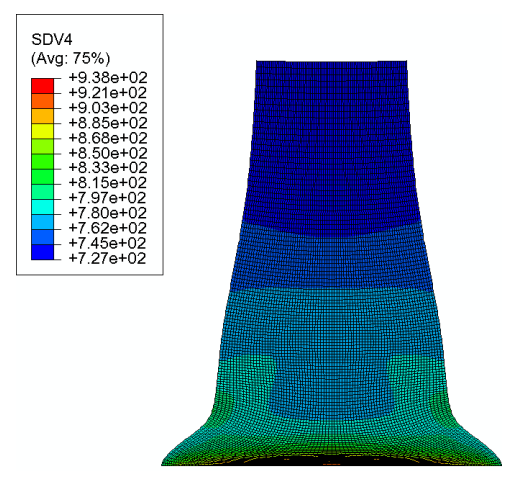

(c) SR-4, temperature [K]

Figure 12: Shot \# 61, temperature at the final time: a) standard MTS model, b) standard PTW model, c) Voce hardening from symbolic regression and expert knowledge on saturation stress

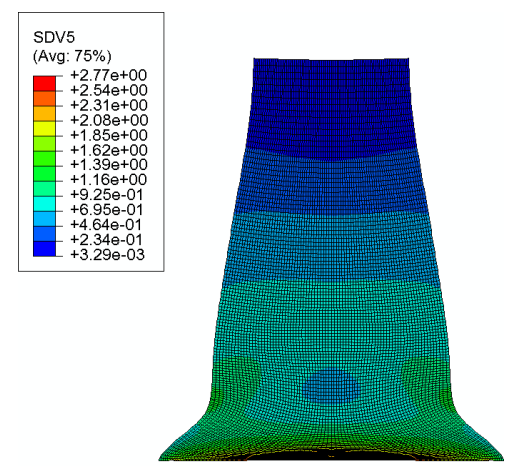

(a) MTS, $\varepsilon^{p}$

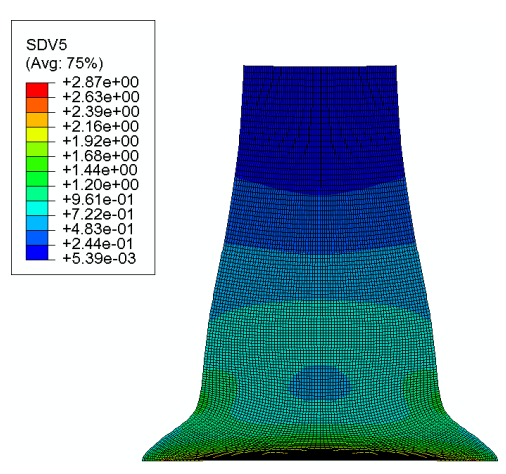

(b) PTW, $\varepsilon^{p}$

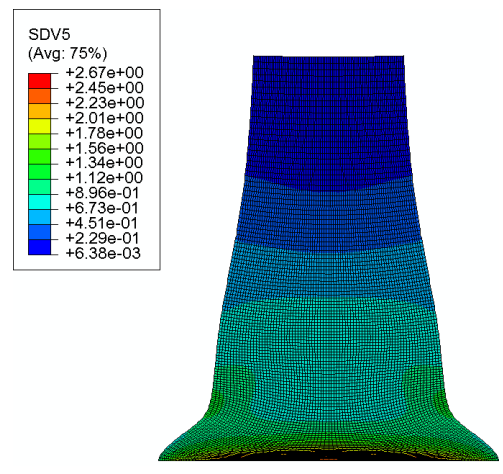

(c) SR-4, $\varepsilon^{p}$

Figure 13: Shot \# 61, equivalent plastic strain at the final time: a) standard MTS model, b) standard PTW model, c) Voce hardening from symbolic regression and expert knowledge on saturation stress 


\section{Conclusions and future works}

While the results obtained with symbolic regression are so far still preliminary, the application of machine learning to modeling flow stress of material under extreme conditions shows great promise, obtaining, in a few trials, models whose performance is comparable to the current state-of-the-art in literature. Besides the quick prototyping, this technique shows several other advantages: it is always possible to guide the learning process by adding a few expertdesignated artificial points to the training dataset, thus avoiding undesirable behavior in parts of the variables' space for which no experimental data is available; and, from a numerical point of view, model development can again be expertly guided by choosing appropriate building blocks, avoiding functions that might introduce excessive numerical issues.

At the same time, as many other machine learning methods, symbolic regression presents clear limits. When no experimental data or expert knowledge is available, the behavior of obtained models is highly unpredictable, and unlikely to be rooted in solid physics. There is also a likelihood of models overfitting the training data, returning good results only for a small part of the variables' space: to avoid this issue, it is necessary to have a representative training set. Moreover, symbolic regression will probably return completely different models for different materials, limiting the re-usability of a result. Additionally, as EAs are stochastic in nature, there is no guarantee that two runs of the algorithm on the same dataset will provide exactly the same results, introducing reproducibility problems. It is important to notice that, while able to satisfyingly predict results, the models found through symbolic regression do not necessarily capture the real physics underlying a phenomenon. Therefore, relevant limitations to extrapolating beyond datasets exist. Further analyses are needed to fully assess the convenience of symbolic regression for problems of this kind. Tests on other materials could provide more information in this regard.

When dealing with white-box machine learning algorithms, where experts can manually review the results, a recurring question is whether or not it is possible for an algorithm to obtain the same expression of a reference human-designed model, for the same phenomenon. For the case study considered in this work, it is almost natural to try and find the common parts between the expressions obtained through symbolic regression and established strength models such as MTS and PTW. Although the question is motivated by the desire of understanding the physics behind the data, it is also true that, from a mathematical point of view, this is a classical ill-posed inverse problem. There is no evidence that the current available strength models, MTS or PTW to mention a few, are the absolutely optimal representation of the physics for this phenomenon, and thus it is possible to find an infinite number of equivalent good approximations of the reality, that ultimately have no common parts. It is also important to notice that human-devised strength models are designed to be as general as possible, while the equations obtained through symbolic regression represent a sort of overfitting on copper data. Again, further experiments on different materials could perhaps provide more evidence to answer this interesting question.

\section{Acknowledgments}

The work of D. Versino and C. A. Bronkhorst at Los Alamos National Laboratory was funded under the Advanced Simulation and Computing (ASC) Program and the Joint DoD/DOE Munitions Technology Development Program (JMP). This support is gratefully acknowledged.

\section{Appendix A.}

Datasets

The datasets used in the machine learning process are taken from the literature. Details of each dataset are reported hereafter, along with their original source. Data points are either taken from tables, or graphically extracted from figures.

\section{Dataset D1}

This first dataset presents six curves, featuring 245-313 points each, for values of $\dot{\varepsilon}^{p}$ varying in $[0.001,0.1,2000] s^{-1}$, and temperatures ranging from $298 \mathrm{~K}$ to $873 \mathrm{~K}[32,45]$. 


\section{Dataset D2}

Dataset D2 is extracted from Figure 3 in [16]. A single experiment is reported, with 25 points at low temperature, $\mathrm{T}=76 \mathrm{~K}$, and $\dot{\varepsilon}^{p}=0.001 s^{-1}$.

\section{Dataset D3}

Dataset D3, from [32], contains strain-stress data for values of $\dot{\varepsilon}^{p}$ ranging from $0.00014 s^{-1}$ to $9500 s^{-1}$, and $\mathrm{T}=295 \mathrm{~K}$. In this case, all the relevant information can be found in Table 1 of the corresponding paper, for a total of seven experiments, with 5-7 points each.

\section{Dataset D4}

Dataset D4 features high values of $\dot{\varepsilon}^{p}\left[8 \cdot 10^{8}, 5.53 \cdot 10^{11}\right] s^{-1}$ at $\mathrm{T}=300 \mathrm{~K}$, taken from [16]. Data is graphically sampled from Figure 9 of the reference, for a total of 10 experiments, with only one point each. It is worth remarking that due to the logarithmic axes employed in the original figure, the data can be affected by large errors.

\section{Dataset D5}

Dataset D5 is found in [46]. Data is graphically extracted from the curves presented in Figures A, B and C of the paper, for a total of 15 experiments with $15-20$ points each. In the reported cases, $\dot{\varepsilon}^{p}$ assumes values ranging from $0.066 \mathrm{~s}^{-1}$ to $2300 \mathrm{~s}^{-1}$, for three values of T: $873 \mathrm{~K}, 1023 \mathrm{~K}$ and $1173 \mathrm{~K}$.

\section{Dataset D6}

Dataset D6 is collected from curves found in [47, 48], in particular from Figures A and B, for a total of 10 experiments, with 8-30 points each. The report, however, presents several important differences in the experimental method with regards to the previous datasets, so after an in-depth analysis, this last block of data was discarded.

\section{Formula building blocks}

The commercial tool used in the ML experiments, Eureqa Formulize, can use different formula building blocks to create candidate equations, as described in Section 2. Building blocks used in the experiments are reported in Table A.3.

Table A.3: Subset of building blocks in Eureqa Formulize that were used during the experiments. W is the arbitrary weight associated to each block that the tool uses to compute the complexity of each candidate solution.

\begin{tabular}{|c|c|}
\hline Building block & $\mathbf{W}$ \\
\hline \multicolumn{2}{|l|}{ Basic } \\
\hline Constant & 1 \\
\hline Integer constant & 1 \\
\hline Addition & 1 \\
\hline Subtraction & 1 \\
\hline Multiplication & 1 \\
\hline Division & 2 \\
\hline Negation & 1 \\
\hline \multicolumn{2}{|l|}{ Other } \\
\hline Minimum & 4 \\
\hline Maximum & 4 \\
\hline
\end{tabular}

\begin{tabular}{|c|c|}
\hline Building block & $\mathbf{W}$ \\
\hline \multicolumn{2}{|l|}{ Trigonometry } \\
\hline Sine & 3 \\
\hline Cosine & 3 \\
\hline Tangent & 4 \\
\hline \multicolumn{2}{|l|}{ Squashing } \\
\hline Logistic func. & 4 \\
\hline Step func. & 4 \\
\hline Sign func. & 4 \\
\hline Gaussian func. & 4 \\
\hline Hyperbolic tan & 4 \\
\hline Error func. & 4 \\
\hline Compl. errf & 4 \\
\hline
\end{tabular}

\begin{tabular}{|c|c|}
\hline Building block & $\mathbf{W}$ \\
\hline \multicolumn{2}{|l|}{ Exponential } \\
\hline Exponential & 4 \\
\hline Natural logarithm & 4 \\
\hline Factorial & 4 \\
\hline Power & 5 \\
\hline Square root & 4 \\
\hline
\end{tabular}

\section{Comparison on the training set}

While the validation of the candidate models on unseen data is illustrated in Section 6, results on the training datasets are reported in this subsection. Table A.4 summarizes the maximum and average relative error per considered dataset. For the $i$-th belonging to an experimental set with numerosity $\mathrm{N}$, the error $e_{i}$ is computed from

$$
e_{i}=\left|\tau_{i}^{m o d e l}-\tau_{i}^{r e f}\right|,
$$


where $\tau^{r e f}$ is the vector collecting the experimental stress and the model is one of the strength models described in the previous sections. Note that MTS is not considered in this appendix because its formulation is based on a history variable. The maximum relative error and average relative error in Table A.4 are respectively given by $\max (\boldsymbol{e}) / \max \left(\boldsymbol{\tau}^{r e f}\right)$ and

$$
\frac{1}{\mathrm{~N}} \sum_{i=1}^{\mathrm{N}} \frac{e_{i}}{\max \left(\tau^{r e f}\right)}
$$

Interestingly, while model SR-1 is often the best on its training datasets, it generalizes poorly for unseen data (dataset D4). From the physical point of view, it confirms that the interpolation of the thermally activated plasticity does not extend to the overdriven-shock regime. Models SR-2, SR-3 and SR-4, on the other hand, provide good results, and often outperform PTW error-wise. Nonetheless, good fit to the training data is not sufficient to ensure good performance on the validation set. In this regard, when SR-2 is compared to SR-3, the former is more accurate on the training set but the latter is the one that performs better on the validation test cases in Section 6. Finally, in Figure A.14 the training dataset used to compute $\tau_{c}$ for SR-3 and SR-4 is shown. This training set is build using data from sets D1 to D5 interpolated at $\varepsilon^{p}=0.23$. As explained in Section 4, SR-4 is obtained from an enriched dataset in order to eliminate the plateau for $\dot{\varepsilon}^{p}>1 \cdot 10^{4} s^{-1}$ that characterizes SR-3.

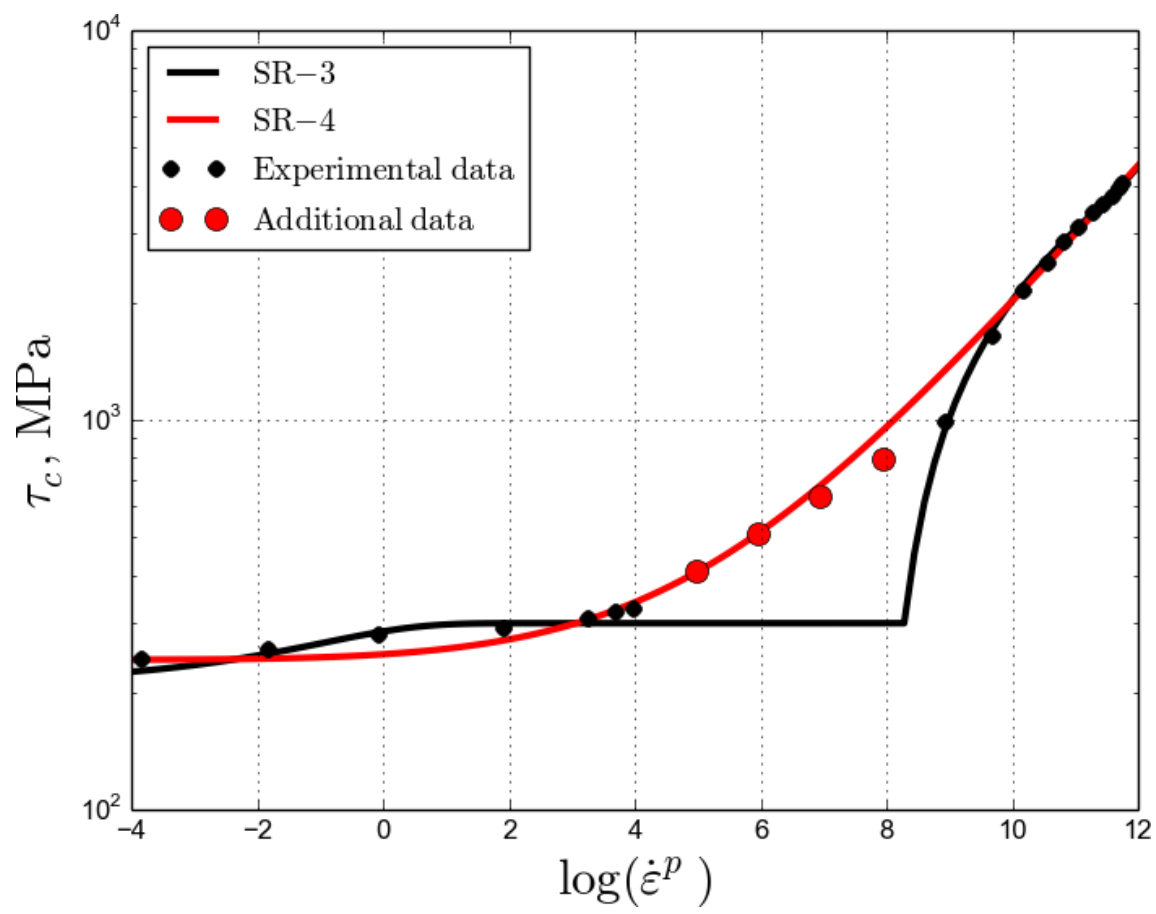

Figure A.14: Comparison of SR-3 and SR-4 with training data for $\varepsilon^{p}=\varepsilon_{f}^{p}=0.23$ and T $=300 \mathrm{~K}$. 
Table A.4: Percent errors, with respect to the experimental data, for the different considered models. For each curve in the datasets the best fitting is highlighted in bold.

\begin{tabular}{|c|c|c|c|c|c|c|c|c|c|c|c|c|}
\hline \multirow[b]{2}{*}{ Set } & \multirow[b]{2}{*}{$\dot{\varepsilon}^{p}\left[s^{-1}\right]$} & \multirow[b]{2}{*}{$\mathrm{T}[\mathrm{K}]$} & \multicolumn{5}{|c|}{ Max percent relative error } & \multicolumn{5}{|c|}{ Average percent relative error } \\
\hline & & & PTW & SR-1 & SR-2 & SR-3 & SR-4 & PTW & SR-1 & SR-2 & SR-3 & SR-4 \\
\hline D1-1 & $2.00 \cdot 10^{3}$ & 298 & 8.32 & 4.34 & 9.03 & 5.65 & 8.39 & 3.94 & 1.19 & 3.82 & 2.60 & 5.20 \\
\hline D1-2 & $2.00 \cdot 10^{3}$ & 473 & 7.23 & 5.10 & 5.32 & 7.56 & 8.04 & 4.01 & 0.92 & 1.94 & 2.13 & 2.58 \\
\hline D1-3 & $1.00 \cdot 10^{-1}$ & 298 & 6.18 & 1.46 & 2.68 & 6.79 & 4.47 & 2.35 & 0.32 & 0.66 & 5.20 & 1.50 \\
\hline D1-4 & $1.00 \cdot 10^{-3}$ & 298 & 3.33 & 0.80 & 2.70 & 4.73 & 5.58 & 1.40 & 0.25 & 0.61 & 1.72 & 2.88 \\
\hline D1-5 & $2.00 \cdot 10^{3}$ & 673 & 10.58 & 7.44 & 7.52 & 14.59 & 13.92 & 7.00 & 2.64 & 2.19 & 2.12 & 2.30 \\
\hline D1-6 & $2.00 \cdot 10^{3}$ & 873 & 14.06 & 8.61 & 5.68 & 23.69 & 22.89 & 7.30 & 1.89 & 1.74 & 3.12 & 2.78 \\
\hline D2-1 & $1.00 \cdot 10^{-3}$ & 76 & 11.94 & 15.84 & 5.97 & 32.82 & 34.50 & 7.16 & 10.68 & 2.77 & 11.21 & 11.53 \\
\hline D3-1 & $1.40 \cdot 10^{-4}$ & 295 & 12.96 & 6.35 & 8.62 & 10.68 & 6.28 & 8.65 & 5.44 & 6.67 & 5.98 & 1.58 \\
\hline D3-2 & $1.50 \cdot 10^{-2}$ & 295 & 14.92 & 10.06 & 17.31 & 21.27 & 23.61 & 7.09 & 6.04 & 6.82 & 5.44 & 7.78 \\
\hline D3-3 & $8.20 \cdot 10^{-1}$ & 295 & 8.35 & 16.60 & 12.04 & 8.15 & 16.40 & 5.68 & 5.49 & 6.69 & 2.70 & 8.87 \\
\hline D3-4 & $8.10 \cdot 10^{1}$ & 295 & 10.93 & 6.43 & 8.40 & 2.65 & 10.73 & 10.34 & 3.27 & 6.29 & 1.60 & 8.27 \\
\hline D3-5 & $1.80 \cdot 10^{3}$ & 295 & 7.42 & 11.25 & 10.45 & 11.85 & 10.21 & 5.39 & 3.89 & 3.87 & 2.99 & 2.23 \\
\hline D3-6 & $5.00 \cdot 10^{3}$ & 295 & 8.05 & 7.39 & 4.14 & 5.55 & 2.80 & 6.61 & 5.77 & 2.48 & 5.14 & 2.37 \\
\hline D3-7 & $9.50 \cdot 10^{3}$ & 295 & 9.20 & 13.06 & 5.51 & 10.97 & 2.88 & 8.41 & 12.24 & 4.61 & 10.12 & 2.38 \\
\hline D4-1 & $8.48 \cdot 10^{8}$ & 300 & 31.82 & 26.59 & 39.80 & 3.68 & 43.91 & 31.82 & 26.59 & 39.80 & 3.68 & 43.91 \\
\hline D4-2 & $4.77 \cdot 10^{9}$ & 300 & 22.88 & 55.56 & 9.46 & 9.14 & 14.19 & 22.88 & 55.56 & 9.46 & 9.14 & 14.19 \\
\hline D4-3 & $1.48 \cdot 10^{10}$ & 300 & 23.67 & 66.31 & 0.57 & 5.96 & 4.07 & 23.67 & 66.31 & 0.57 & 5.96 & 4.07 \\
\hline D4-4 & $3.51 \cdot 10^{10}$ & 300 & 30.54 & 71.35 & 2.31 & 5.40 & 2.13 & 30.54 & 71.35 & 2.31 & 5.40 & 2.13 \\
\hline D4-5 & $6.36 \cdot 10^{10}$ & 300 & 34.30 & 74.59 & 4.03 & 2.99 & 0.05 & 34.30 & 74.59 & 4.03 & 2.99 & 0.05 \\
\hline D4-6 & $1.09 \cdot 10^{11}$ & 300 & 40.66 & 76.75 & 3.39 & 2.45 & 0.31 & 40.66 & 76.75 & 3.39 & 2.45 & 0.31 \\
\hline D4-7 & $1.88 \cdot 10^{11}$ & 300 & 46.55 & 78.84 & 3.05 & 1.13 & 0.09 & 46.55 & 78.84 & 3.05 & 1.13 & 0.09 \\
\hline D4-8 & $2.74 \cdot 10^{11}$ & 300 & 54.50 & 79.70 & 0.31 & 2.66 & 2.41 & 54.50 & 79.70 & 0.31 & 2.66 & 2.41 \\
\hline D4-9 & $3.79 \cdot 10^{11}$ & 300 & 59.86 & 80.64 & 1.05 & 2.94 & 3.30 & 59.86 & 80.64 & 1.05 & 2.94 & 3.30 \\
\hline D4-10 & $4.96 \cdot 10^{11}$ & 300 & 63.17 & 81.53 & 1.45 & 2.51 & 3.24 & 63.17 & 81.53 & 1.45 & 2.51 & 3.24 \\
\hline D5-1 & $6.60 \cdot 10^{-2}$ & 873 & 24.16 & 187.87 & 34.79 & 67.05 & 82.07 & 21.40 & 74.43 & 22.26 & 45.60 & 60.40 \\
\hline D5-2 & $9.60 \cdot 10^{2}$ & 873 & 18.29 & 28.10 & 21.88 & 29.71 & 22.40 & 11.93 & 13.63 & 11.08 & 12.25 & 5.81 \\
\hline D5-3 & $1.25 \cdot 10^{3}$ & 873 & 14.40 & 25.36 & 20.58 & 23.84 & 18.86 & 10.90 & 14.52 & 11.32 & 9.59 & 5.51 \\
\hline D5-4 & $1.80 \cdot 10^{3}$ & 873 & 12.49 & 30.01 & 22.00 & 13.51 & 11.80 & 9.21 & 13.76 & 9.02 & 4.56 & 3.24 \\
\hline D5-5 & $2.30 \cdot 10^{3}$ & 873 & 12.39 & 31.57 & 21.30 & 5.06 & 5.61 & 10.76 & 15.75 & 6.66 & 2.41 & 2.61 \\
\hline D5-6 & $6.60 \cdot 10^{-2}$ & 1023 & 19.18 & 430.90 & 45.55 & 57.40 & 80.40 & 16.96 & 158.80 & 29.08 & 37.23 & 56.73 \\
\hline D5-7 & $9.60 \cdot 10^{2}$ & 1023 & 20.03 & 53.69 & 15.32 & 18.53 & 9.91 & 16.53 & 19.01 & 5.43 & 9.80 & 5.86 \\
\hline D5-8 & $1.25 \cdot 10^{3}$ & 1023 & 19.98 & 67.18 & 19.60 & 15.83 & 10.63 & 17.37 & 24.95 & 6.17 & 8.68 & 6.77 \\
\hline D5-9 & $1.80 \cdot 10^{3}$ & 1023 & 18.52 & 79.44 & 22.46 & 14.80 & 13.18 & 14.57 & 33.33 & 7.30 & 9.33 & 8.57 \\
\hline D5-10 & $2.30 \cdot 10^{3}$ & 1023 & 18.13 & 83.45 & 22.99 & 12.84 & 14.26 & 12.84 & 39.15 & 8.52 & 8.63 & 9.39 \\
\hline D5-11 & $6.60 \cdot 10^{-2}$ & 1173 & 5.85 & 866.76 & 55.34 & 16.09 & 31.81 & 4.23 & 322.75 & 34.73 & 9.82 & 25.79 \\
\hline D5-12 & $9.60 \cdot 10^{2}$ & 1173 & 21.57 & 115.90 & 11.20 & 21.54 & 10.56 & 19.81 & 36.15 & 6.29 & 11.61 & 6.48 \\
\hline D5-13 & $1.25 \cdot 10^{3}$ & 1173 & 23.60 & 137.41 & 14.48 & 19.96 & 13.24 & 19.33 & 46.77 & 8.81 & 11.37 & 8.38 \\
\hline D5-14 & $1.80 \cdot 10^{3}$ & 1173 & 22.68 & 171.00 & 21.39 & 18.36 & 17.62 & 16.13 & 65.53 & 11.91 & 11.74 & 11.30 \\
\hline D5-15 & $2.30 \cdot 10^{3}$ & 1173 & 19.12 & 169.03 & 24.40 & 16.47 & 19.85 & 13.64 & 75.16 & 14.60 & 11.01 & 13.33 \\
\hline D1, ave & & & 8.28 & 4.62 & 5.49 & 10.50 & 10.55 & 4.33 & 1.20 & 1.83 & 2.81 & 2.87 \\
\hline D2, ave & & & 11.94 & 15.84 & 5.97 & 32.82 & 34.50 & 7.16 & 10.68 & 2.77 & 11.21 & 11.53 \\
\hline D3, ave & age & & 10.26 & 10.16 & 9.50 & 10.16 & 10.42 & 7.45 & 6.02 & 5.35 & 4.85 & 4.78 \\
\hline D4, ave & age & & 40.80 & 69.19 & 6.54 & 3.89 & 7.37 & 40.80 & 69.19 & 6.54 & 3.89 & 7.37 \\
\hline D5, ave & age & & 18.02 & 165.18 & 24.89 & 23.40 & 24.15 & 14.37 & 63.58 & 12.88 & 13.58 & 15.34 \\
\hline
\end{tabular}




\section{References}

[1] J. R. Koza, Genetic Programming: vol. 1, On the programming of computers by means of natural selection, Vol. 1, MIT press, 1992.

[2] A. S. Argon, Physics of strength and plasticity, MIT press, 1969.

[3] U. F. Kocks, A. S. Argon, A. M. F., Thermodynamics and kinetics of slip, Pergamon press, 1975.

[4] A. S. Argon, Constitutive equations in plasticity, MIT press, 1975.

[5] P. S. Follansbee, Fundamentals of strength: principles, experiment, and applications of an internal state variable constitutive formulation, John Wiley \& Sons, 2014

[6] U. F. Kocks, C. N. Tome, H.-R. Wenk, Texture and anisotropy, Cambridge University Press, 1998.

[7] A. S. Argon, Strengthening mechanisms in crystal plasticity, Oxford University Press, 2006.

[8] L. Priester, Grain boundaries and crystalline plasticity, John Wiley \& Sons, 2011.

[9] M. E. Gurtin, E. Fried, L. Anand, The mechanics and thermodynamics of continua, Cambridge University press, 2010.

[10] L. P. Kubin, Dislocations, mesoscale simulations and plastic flow, Oxford University press, 2013.

[11] R. Bobbili, B. Ramakrishna, V. Madhu, A. Gogia, Prediction of flow stress of 7017 aluminium alloy under high strain rate compression at elevated temperatures, Defence Technology 11 (1) (2015) $93-98$.

[12] H.-Y. Li, X.-F. Wang, D.-D. Wei, J.-D. Hu, Y.-H. Li, A comparative study on modified zerilli-armstrong, arrhenius-type and artificial neural network models to predict high-temperature deformation behavior in t24 steel, Materials Science and Engineering: A 536 (2012) $216-222$.

[13] A. Asgharzadeh, H. Jamshidi Aval, S. Serajzadeh, A study on flow behavior of aa5086 over a wide range of temperatures, Journal of Materials Engineering and Performance 25 (3) (2016) 1076-1084.

[14] S. K. Singh, R. K. Desu, S. C. Guntuku, A. B, A. K. Gupta, Support vector regression based flow stress prediction in austenitic stainless steel 304, in: 3rd International Conference on Materials Processing and Characterisation (ICMPC 2014), Vol. 6, 2014 , pp. 368 - 375.

[15] G. Taylor, The use of flat-ended projectiles for determining dynamic yield stress. I. theoretical considerations, Proceedings of the Royal Society of London A: Mathematical, Physical and Engineering Sciences 194 (1038) (1948) 289-299.

[16] D. L. Preston, D. L. Tonks, D. C. Wallace, Model of plastic deformation for extreme loading conditions, Journal of Applied Physics 93 (1) (2003) 211-220.

[17] E. Buckingham, On physically similar systems; illustrations of the use of dimensional equations, Phys. Rev. 4 (1914) 345-376.

[18] K. A. De Jong, Evolutionary computation: a unified approach, MIT press, 2006.

[19] M. Schmidt, H. Lipson, Distilling free-form natural laws from experimental data, Science 324 (5923) (2009) 81-85.

[20] M. D. Schmidt, H. Lipson, Coevolution of fitness predictors, IEEE Transactions on Evolutionary Computation 12 (6) (2008) $736-749$.

[21] M. D. Schmidt, H. Lipson, Incorporating expert knowledge in evolutionary search: a study of seeding methods, in: Proceedings of the 11th Annual conference on Genetic and evolutionary computation, ACM, 2009, pp. 1091-1098.

[22] M. D. Schmidt, H. Lipson, Learning noise, in: Proceedings of the 9th Annual Conference on Genetic and Evolutionary Computation, GECCO '07, ACM, New York, NY, USA, 2007, pp. 1680-1685.

[23] M. Schmidt, H. Lipson, Age-Fitness Pareto Optimization, Springer New York, New York, NY, 2011, pp. 129-146.

[24] G. Rudolph, Convergence analysis of canonical genetic algorithms, IEEE transactions on neural networks 5 (1) (1994) 96-101.

[25] J. D. Lohn, G. S. Hornby, A. Rodriguez-Arroyo, D. S. Linden, W. F. Kraus, S. E. Seufert, Evolutionary design of an X-band antenna for NASA's space technology 5 mission, Tech. rep., NASA (2003).

[26] E. Sanchez, G. Squillero, A. Tonda, Industrial applications of evolutionary algorithms, Vol. 34, Springer, 2012.

[27] K. Stanislawska, K. Krawiec, Z. W. Kundzewicz, Modeling global temperature changes with genetic programming, Comput. Math. Appl. 64 (12) (2012) 3717-3728.

[28] G. Johnson, W. Cook, A constitutive model and data for metals subjected to large strains, high strain rates, and high temperatures, in: Proc. 7th International Symp. on Ballistics, 1983.

[29] D. J. Steinberg, S. G. Cochran, M. W. Guinan, A constitutive model for metals applicable at highstrain rate, Journal of Applied Physics 51 (3) (1980) 1498-1504.

[30] D. J. Steinberg, C. M. Lund, A constitutive model for strain rates from $10^{-4}$ to $10^{6} \mathrm{~s}^{-1}$, Journal of Applied Physics 65 (4) (1989) $1528-1533$.

[31] F. J. Zerilli, R. W. Armstrong, Dislocationmechanicsbased constitutive relations for material dynamics calculations, Journal of Applied Physics 61 (5) (1987) 1816-1825.

[32] P. Follansbee, U. Kocks, A constitutive description of the deformation of copper based on the use of the mechanical threshold stress as an internal state variable, Acta Metallurgica 36 (1) (1988) 81-93.

[33] H. M. Mourad, C. A. Bronkhorst, F. L. Addessio, C. M. Cady, D. W. Brown, S. R. Chen, G. T. Gray III, Incrementally objective implicit integration of hypoelastic-viscoplastic constitutive equations based on the mechanical threshold strength model, Computational Mechanics 53 (5) (2014) 941-955.

[34] U. Kocks, Laws for work-hardening and low-temperature creep, Journal of engineering materials and technology 98 (1) (1976) $76-85$.

[35] P. Follansbee, G. Gray, An analysis of the low temperature, low and high strain-rate deformation of Ti-6Al-4V, Metallurgical Transactions A 20 (5) (1989) 863-874.

[36] P. Follansbee, J. Huang, G. Gray, Low-temperature and high-strain-rate deformation of nickel and nickel-carbon alloys and analysis of the constitutive behavior according to an internal state variable model, Acta Metallurgica et Materialia 38 (7) (1990) 1241-1254.

[37] D. C. Wallace, Irreversible thermodynamics of overdriven shocks in solids, Physical Review B 24 (10) (1981) 5597.

[38] ABAQUS, Abaqus Theory Manual, Dassault Systémes Simulia Corp, Providence (2011).

[39] J. Simo, T. Hughes, Computational Inelasticity, Springer New York, 1998.

[40] D. J. Luscher, C. A. Bronkhorst, D. L. McDowell, Effects of local and nonlocal substructure spin on localization in tantalum tophat specimen, Technische Mechanik 32 (2-5) (2012) 393-407.

[41] M. A. Zocher, P. J. Maudlin, S. R. Chen, E. C. Flower-Maudlin, An evaluation of several hardening models using taylor cylinder impact data, European Congress on Computational Methods in Applied Sciences and Engineering, Barcelona, Spain, 2000.

[42] B. Holmedal, On the formulation of the mechanical threshold stress model, Acta Materialia 55 (8) (2007) $2739-2746$. 
[43] W. H. Gust, High impact deformation of metal cylinders at elevated temperatures, Journal of Applied Physics 53 (5) (1982) $3566-3575$.

[44] L. Burakovsky, D. L. Preston, R. R. Silbar, Analysis of dislocation mechanism for melting of elements: Pressure dependence, Journal of Applied Physics 88 (11) (2000) 6294-6301.

[45] S. R. Chen, G. T. Gray, Mechanical properties and constitutive modeling of metals, Tech. Rep. LA-UR-09-07780 (2009).

[46] S. Samanta, Dynamic deformation of aluminium and copper at elevated temperatures, Journal of the Mechanics and Physics of Solids 19 (3) (1971) 117-135.

[47] B. Banerjee, An evaluation of plastic flow stress models for the simulation of high-temperature and high-strain-rate deformation of metals, arXiv preprint cond-mat/0512466

[48] B. Banerjee, The mechanical threshold stress model for various tempers of aisi 4340 steel, International Journal of Solids and Structures 44 (3-4) (2007) 834-859. 